CERN-TH/2002-170

MC-TH-2002-03

hep-ph/0207277

July 2002

\title{
Higgs-Mediated Electric Dipole Moments in the MSSM: An Application to Baryogenesis and Higgs Searches
}

\author{
Apostolos Pilaftsis ${ }^{a, b}$ \\ a Theory Division, CERN, CH-1211 Geneva 23, Switzerland \\ ${ }^{b}$ Department of Physics and Astronomy, University of Manchester, \\ Manchester M13 9PL, United Kingdom
}

\begin{abstract}
We perform a comprehensive study of the dominant two- and higher-loop contributions to the ${ }^{205} \mathrm{Tl}$, neutron and muon electric dipole moments induced by Higgs bosons, thirdgeneration quarks and squarks, charginos and gluinos in the Minimal Supersymmetric Standard Model (MSSM). We find that strong correlations exist among the contributing CP-violating operators, for large stop, gluino and chargino phases, and for a wide range of values of $\tan \beta$ and charged Higgs-boson masses, giving rise to large suppressions of the ${ }^{205} \mathrm{Tl}$ and neutron electric dipole moments below their present experimental limits. Based on this observation, we discuss the constraints that the non-observation of electric dipole moments imposes on the radiativelygenerated CP-violating Higgs sector and on the mechanism of electroweak baryogenesis in the MSSM. We improve previously suggested benchmark scenarios of maximal CP violation for analyzing direct searches of CP-violating MSSM Higgs bosons at high-energy colliders, and stress the important complementary rôle that a possible high-sensitivity measurement of the muon electric dipole moment to the level of $10^{-24} e \mathrm{~cm}$ can play in such analyses.
\end{abstract}

PACS numbers: 11.30.Er, 14.80.Er 


\section{Introduction}

The non-observation of electric dipole moments (EDMs) of the thallium atom and neutron, as well as the absence of large flavour-changing neutral-current (FCNC) decays put severe constraints on the parameters of a theory. Especially, these constraints become even more severe for supersymmetric theories, such as the MSSM, in which too large FCNC and CPviolating effects are generically predicted at the one-loop level, resulting in gross violations with experimental data. A possible resolution of such FCNC and CP crises, often considered in the literature [1], makes use of the decoupling properties of the heavy squarks and sleptons of the first two generations, whose masses should be larger than $\sim 10 \mathrm{TeV}$. Thus, for sufficiently heavy squarks and sleptons, the one-loop predictions for FCNC and EDM observables can be suppressed up to levels compatible with experiment. Also, such a solution poses no serious problem to the gauge hierarchy, as long as the first two generations of squarks and sleptons are not much heavier than $10 \mathrm{TeV}$. In this case, because of their suppressed Yukawa couplings, the radiative effect of the first two generations of sfermions on the Higgs-boson mass spectrum is still negligible, with respect to that of TeV scalar top and bottom quarks.円

Recently, it has been shown [2, 3] that even third-generation squarks may lead by themselves to observable effects on the electron and neutron EDMs through Higgs-bosonmediated two-loop graphs of the Barr-Zee type [4. This observation offers new possibilities to probe the CP-violating soft-supersymmetry-breaking parameters related to the thirdgeneration squarks. Most interestingly, the same CP-violating parameters may induce radiatively a CP-noninvariant Higgs-sector [5, 6, 7, 8], leading to novel signatures at highenergy colliders [8, 9, 10, 11]. It is then obvious that EDM constraints do have important implications for the phenomenological predictions within the above framework of the MSSM with explicit CP violation. Moreover, employing upper limits on EDMs, one is, in principle, able to derive constraints on the phase of the $\mathrm{SU}(2)_{L}$ gaugino mass, $m_{\widetilde{W}}$, which plays a central rôle in electroweak baryogenesis [12] in the MSSM [13, 14].

On the experimental side, the current upper limit on the electron EDM $d_{e}$, as derived from the absence of a permanent atomic EDM for ${ }^{205} \mathrm{Tl}$, has improved by a factor of almost 2 over the last few years [15, 16]. Specifically, the reported $2 \sigma$ upper limit on a thallium EDM is [16]

$$
\left|d_{\mathrm{Tl}}\right| \lesssim 1.3 \times 10^{-24} e \mathrm{~cm} .
$$

\footnotetext{
${ }^{1}$ One should bear in mind that radiative effects on the neutral Higgs-boson masses are proportional to the fourth power of Yukawa couplings. A simple estimate indicates that the contribution of the second generation of sfermions is smaller, by a factor of at least $10^{-7}$, than those of the third generation.
} 
Then, the electron EDM $d_{e}$ may be deduced indirectly by means of the effective Lagrangian

$$
\begin{aligned}
\mathcal{L}_{\mathrm{EDM}}= & -\frac{1}{2} d_{e} \bar{e} \sigma_{\mu \nu} i \gamma_{5} e F^{\mu \nu}+C_{S} \bar{N} N \bar{e} i \gamma_{5} e+C_{P} \bar{N} i \gamma_{5} N \bar{e} e \\
& +C_{T} \bar{N} \sigma_{\mu \nu} i \gamma_{5} N \bar{e} \sigma^{\mu \nu} e+\ldots,
\end{aligned}
$$

where $C_{S}, C_{P}, C_{T}$ and the ellipses denote $\mathrm{CP}$-violating operators of dimension 6 and higher. With the aid of the effective Lagrangian (1.2), the atomic EDM of ${ }^{205} \mathrm{Tl}$ may be computed by [17, 18, 19]

$$
\begin{aligned}
d_{\mathrm{Tl}}[e \mathrm{~cm}]= & -585 \times d_{e}[e \mathrm{~cm}]+8.5 \times 10^{-19}[e \mathrm{~cm}] \times C_{S}\left[\mathrm{TeV}^{-2}\right] \\
& -8 . \times 10^{-22}[e \mathrm{~cm}] \times C_{T}\left[\mathrm{TeV}^{-2}\right]+\ldots
\end{aligned}
$$

In (1.3), the dots denote CP-odd operators of dimension 7 and higher. In our analysis, we will assume that like $C_{T}$, the CP-odd operators of dimension 7 and higher give rise generically to negligible effects on the ${ }^{205} \mathrm{Tl} \mathrm{EDM}$. Moreover, although the contributions of the neglected CP-odd operators to other heavy atoms may be comparable to that of $d_{e}$, the experimental upper limits are still much weaker than $d_{\mathrm{Tl}}$, by at least one order of magnitude. Consequently, we will only analyze predictions for the thallium EDM $d_{\mathrm{Tl}}$ and consider only two operators: the electron EDM $d_{e}$ and the CP-odd electron-nucleon operator $C_{S}$. From (1.1) and (1.3), it is then not difficult to deduce the following $2 \sigma$ upper limits on these two CP-odd operators:

$$
\left|d_{e}\right| \lesssim 2.2 \times 10^{-27} \text { e cm }, \quad\left|C_{S}\right| \lesssim 1.5 \times 10^{-6}\left[\mathrm{TeV}^{-2}\right]
$$

In the MSSM under study, the contributions from $d_{e}$ and $C_{S}$ to $d_{\mathrm{Tl}}$ can be of comparable size and therefore cannot be treated independently. In fact, depending on their relative sign, one may increase or reduce the EDM bounds on the CP-violating parameters of the theory. Here, the proposed high-sensitivity measurement of the muon EDM $d_{\mu}$ to the level $10^{-24} e \mathrm{~cm}[20]$ may offer new constraints complementary to those obtained by $d_{\mathrm{Tl}}$, since $C_{S}$ and all higher-dimensional CP-odd operators are absent.

Unlike the thallium EDM, the upper limit on the neutron EDM $d_{n}$ is less severe, i.e.

$$
\left|d_{n}\right| \lesssim 1.2 \times 10^{-25} \mathrm{ecm}
$$

at the $2 \sigma$ confidence level (CL) [21, 22, 23]. Moreover, although promising computations based on QCD sum rules [24] appeared recently, the theoretical prediction for $d_{n}$ is rather model-dependent. For example, the predictions between the valence-quark and quark-parton models may differ, even up to one order of magnitude [25]. Recently, the 
experimental upper limit on a permanent EDM of the ${ }^{199} \mathrm{Hg}$ atom has been improved by a factor of 4, i.e. $\left|d_{\mathrm{Hg}}\right|<2.33 \times 10^{-28} \mathrm{e} \mathrm{cm}$ at the $2 \sigma \mathrm{CL}$ [26]. On the theoretical side, however, the derivation of bounds [27] from $d_{\mathrm{Hg}}$ on the chromoelectric dipole moment (CEDM) operators of $u$ and $d$ quarks contains many uncertainties related to unknown effects of higher-dimensional chiral operators, nucleon-current ambiguities [28], the neglect of the CP-odd three-gluon operator [29, 30], the modelling for extracting the nuclear Schiff moment [19], the $s$-quark content in heavy nuclei, etc. Thus, we shall not implement mercury EDM constraints in our analysis. Instead, we will consider that no large cancellations [31] below the $10 \%$ level occur among the different EDM terms in the neutron EDM. In a sense, such a procedure takes account of a possible complementarity relation [27] between the measurements of the neutron and Hg EDMs.

As we have already mentioned above, in the MSSM one-loop EDM effects 32, 33, 34, 25] can be greatly suppressed below their experimental limits, if the first two generation of squarks and sleptons are made heavy enough, typically heavier than $10 \mathrm{TeV}$ [33, 25]. Within such a framework of the MSSM [35], the dominant contributions to EDMs arise from Higgs-mediated Barr-Zee-type two-loop graphs that involve quarks and squarks of the third generation, charginos and gluinos. [n this paper, we improve previous computations of these two-loop contributions to EDMs, by resumming CP-even and CP-odd radiative effects on the Higgs-boson self-energies and vertices 38, 39. Analogous improvements of higher-order resummation effects are also considered in the computation of the CP-odd electron-nucleon operator $C_{S}$. Within the above resummation approach, we compute the original Barr-Zee EDM graph induced by $t$-quarks beyond the two-loop approximation in the MSSM through one-loop CP-violating threshold corrections to the top-quark Yukawa coupling. Finally, we compute the Higgs-boson two-loop contribution to EDM induced by charginos, and discuss the consequences of the derived EDM constraints on electroweak baryogenesis in the MSSM.

The present paper is organized as follows: in Section 2, we discuss the CP-odd electron-nucleon operator $C_{S}$, which gives an enhanced contribution to the thallium EDM

\footnotetext{
${ }^{3}$ Alternatively, one-loop EDM contributions can be suppressed if the CP phases of the trilinear softYukawa couplings of the first two generations and the CP phases of Wino $\tilde{W}$, Bino $\tilde{B}$ and gluino $\tilde{g}$ are all zero, with $B \mu$ and $\mu$ being positive according to our CP conventions. In this case, however, if the first two generations of sfermions are relatively light, e.g. few hundreds of GeV, then additional two-loop EDM graphs [36] exist, such as those induced by a gluino CEDM, which give non-negligible contributions to the EDMs. Furthermore, there are two-loop EDM effects induced by a CP-odd $\gamma W^{+} W^{-}$operator, which do not decouple in the limit of heavy squarks and do not depend on Higgs-boson masses [37. These two-loop EDM contributions are subdominant, yielding an electron EDM term typically smaller than $10^{-27} e \mathrm{~cm}$.
} 
$d_{\mathrm{Tl}}$ in the large $\tan \beta$ regime [40]. In Section 3, after reviewing the existing dominant Higgs-boson two-loop contributions to EDMs, we compute the very relevant Barr-Zee contribution to EDM from $t$ quarks for the first time in the MSSM. In addition, we critically re-examine a very recent calculation [41] on Higgs-boson two-loop EDM effects due to charginos. In Sections 2 and 3, we also improve previous computations of the CP-odd electron-nucleon operator $C_{S}$ and the electron EDM $d_{e}$, by taking properly into account higher-order CP-even and CP-odd resummation effects of Higgs-boson self-energy and vertex graphs. Section 4 is devoted to numerical estimates of EDMs and discusses the impact of the derived EDM constraints on electroweak baryogenesis and on the analysis of direct searches for CP-violating Higgs bosons. Our conclusions are drawn in Section 5.

\section{CP-odd electron-nucleon operator $C_{S}$}

Let us first study the contribution of the CP-odd electron-nucleon operator $C_{S}$ [17, 18, 19] to the ${ }^{205} \mathrm{Tl}$ EDM. At the elementary particle level, $C_{S}$ can be induced by two types of CP-odd operators in supersymmetric theories: $\bar{e} i \gamma_{5} e \bar{q} q$ [40 and $\bar{e} i \gamma_{5} e \tilde{q}^{*} \tilde{q}$, where $q$ and $\tilde{q}$ denote quark and squark fields, respectively. In the MSSM, the above two CP-odd operators of dimensions 6 and 5 are generated by interactions involving Higgs scalar-pseudoscalar mixing and CP-violating vertex effects, as those shown in Fig. 11.

However, not all quarks and squarks can give rise to potentially large contributions to the ${ }^{205} \mathrm{Tl}$ EDM. Our interest is to consider only enhanced Yukawa and trilinear couplings of the Higgs bosons to quarks and squarks in the decoupling limit of the first two generation of squarks. This criterion singles out the CP-odd operators related to top and bottom quarks, and their supersymmetric partners. In fact, as is shown in Fig. 1, heavy quarks and squarks do not contribute directly to the CP-odd operator $C_{S}$, but only through the loop-induced Higgs-gluon-gluon couplings $H_{i} g g$, after they have been integrated out. Thus, the effective Lagrangian responsible for generating $C_{S}$ is

$$
\mathcal{L}_{\mathrm{eff}}^{\left(C_{S}\right)}=\sum_{i=1}^{3} \frac{g_{w} H_{i}}{2 M_{W}}\left(g_{H_{i} g g} \frac{\alpha_{s}}{8 \pi} G^{a, \mu \nu} G_{\mu \nu}^{a}+m_{e} \tan \beta O_{3 i} \bar{e} i \gamma_{5} e\right),
$$

where $M_{W}=g_{w} v / 2, O$ is the $3 \times 3$-mixing matrix that relates the weak to mass eigenstates of the CP-violating Higgs bosons [6, 8], and

$$
g_{H_{i} g g}=\sum_{q=t, b}\left\{\frac{2}{3} g_{H_{i} q q}^{S}+\frac{v^{2}}{6 m_{\tilde{q}_{1}}^{2} m_{\tilde{q}_{2}}^{2}}\left[\left(m_{\tilde{q}_{2}}^{2}-m_{\tilde{q}_{1}}^{2}\right) \xi_{q}^{\left(H_{i}\right)}+\left(m_{\tilde{q}_{1}}^{2}+m_{\tilde{q}_{2}}^{2}\right) \zeta_{q}^{\left(H_{i}\right)}\right]\right\} .
$$




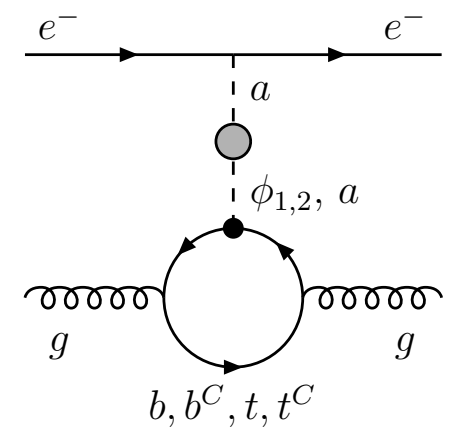

(a)

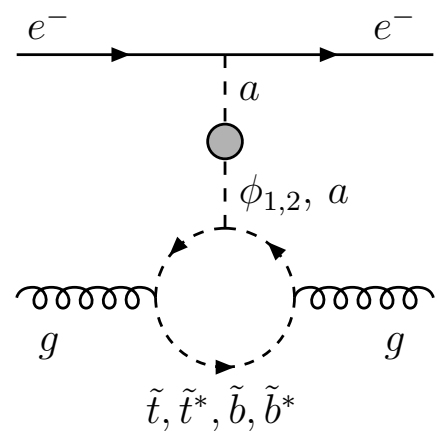

(b)

Figure 1: Feynman graphs contributing to a non-vanishing CP-odd electron-nucleon operator $C_{S}$. At the elementary particle level, $C_{S}$ is predominantly induced by quantum effects involving (a) $t-, b$ - quarks and (b) $\tilde{t}-, \tilde{b}$ - squarks. Blobs and heavy dots denote resummation of self-energy and vertex graphs, respectively.

In (2.2), the dimensionless coefficients $g_{H_{i} q q}^{S}, \xi_{q}^{\left(H_{i}\right)}, \zeta_{q}^{\left(H_{i}\right)}$ and the stop and sbottom masses are given in the appendix.

The largest contribution to the coupling parameter $g_{H_{i} g g}$ comes from the scalar part of the $H_{i} \bar{b} b$ coupling, $g_{H_{i} b b}^{S}$. More explicitly, there are two CP-violating effects that dominate $g_{H_{i} b b}^{S}$ : (i) the $\tan ^{2} \beta$-enhanced threshold effects [40] described by the term

$$
g_{H_{i} b b}^{S} \sim \operatorname{Im}\left[\frac{\left(\Delta h_{b} / h_{b}\right) \tan ^{2} \beta}{1+\left(\delta h_{b} / h_{b}\right)+\left(\Delta h_{b} / h_{b}\right) \tan \beta}\right] O_{3 i},
$$

and (ii) the scalar-pseudoscalar mixing effects contained in the mixing matrix elements $O_{1 i}$. The definition of the quantities $\delta h_{b} / h_{b}$ and $\Delta h_{b} / h_{b}$ may be found in the appendix.

At this stage, it is important to observe that if $\left(\Delta h_{b} / h_{b}\right) \tan \beta \gtrsim 1$, the $\tan ^{2} \beta$ dependence of the CP-violating threshold effects on $g_{H_{i} b b}^{S}$ and $g_{H_{i} b b}^{P}$ considerably modifies. In particular, in the large $\tan \beta$ limit, $g_{H_{i} b b}^{S}$ and $g_{H_{i} b b}^{P}$ asymptotically approach a $\tan \beta$ independent constant, i.e.

$$
\begin{aligned}
g_{H_{i} b b}^{S} & \rightarrow \operatorname{Im}\left[\frac{1+\left(\delta h_{b} / h_{b}\right)}{\left(\Delta h_{b} / h_{b}\right)}\right] O_{3 i}, \\
g_{H_{i} b b}^{P} & \rightarrow \operatorname{Im}\left[\frac{1+\left(\delta h_{b} / h_{b}\right)}{\left(\Delta h_{b} / h_{b}\right)}\right] O_{1 i} .
\end{aligned}
$$

Although the above limits may only be attainable in a very large $\tan \beta$ and quasinonperturbative regime of the theory, the onset of a $\tan \beta$-independent behaviour in $g_{H_{i} b b}^{S}$ 
and $g_{H_{i} b b}^{P}$ may already start from moderately large values of $\tan \beta$, i.e. for $\tan \beta \gtrsim 30$. Consequently, the limits (2.4) and (2.5) should be regarded as upper bounds on the CP-violating threshold-enhanced parts of the coupling parameters $g_{H_{i} b b}^{S}$ and $g_{H_{i} b b}^{P}$. In our numerical analysis in Section 4, we properly take into account the above-described CP-violating resummation effects on $g_{H_{i} b b}^{S}$.

The computation of the CP-odd electron-nucleon operator $C_{S}$ can now be performed by utilizing standard QCD techniques based on the trace anomaly of the energy-momentum tensor [42]. In the chiral quark mass limit, we then have the simple relation

$$
\left\langle N\left|\frac{\alpha_{s}}{8 \pi} G^{a, \mu \nu} G_{\mu \nu}^{a}\right| N\right\rangle=-(100 \mathrm{MeV}) \bar{N} N .
$$

With the help of (2.6), we can evaluate the effective $H_{i} \bar{N} N$ couplings, and hence the CP-odd operator $C_{S}$ :

$$
C_{S}=-(0.1 \mathrm{GeV}) \tan \beta \frac{m_{e} \pi \alpha_{w}}{M_{W}^{2}} \sum_{i=1}^{3} \frac{g_{H_{i} g g} O_{3 i}}{M_{H_{i}}^{2}} .
$$

Observe that the operator $C_{S}$ exhibits an enhanced $\tan ^{3} \beta$ dependence [40]; it therefore becomes very significant for intermediate and large values of $\tan \beta$. Numerical estimates for this contribution to a thallium EDM will be presented in Section 4.

\section{Higgs-boson two-loop contributions to $d_{e}$}

We now turn our attention to Higgs-boson two-loop effects [2, 3] on the electron EDM analogous to those first discussed by Barr and Zee «1 in non-supersymmetric theories. As is shown in Fig. 2, these two-loop EDM effects originate predominantly from graphs that involve: stop and sbottom squarks (Fig. 2(a,b)) [2], top and bottom quarks (Fig. 2(c)), and charginos (Fig. 2(d)) 41].

Strictly speaking, the original Barr-Zee graphs induced by top and bottom quarks in Fig. 2(c) appear beyond the two-loop approximation in the MSSM. However, it is a formidable task to analytically compute the complete set of the relevant three- and higherloop graphs. Therefore, we consider only a subset of higher-loop corrections, in which the dominant CP-violating terms in the Higgs-boson propagators and the Higgs-quark-quark vertices are resummed. Such an approach should only be viewed as an effective one, which is expected to capture the main bulk of the higher-order effects. In the same vein, we improve previous two-loop EDM calculations related to third-generation squarks [2] and charginos 41] by resumming dominant CP-violating self-energy terms in the Higgs-boson propagators. 


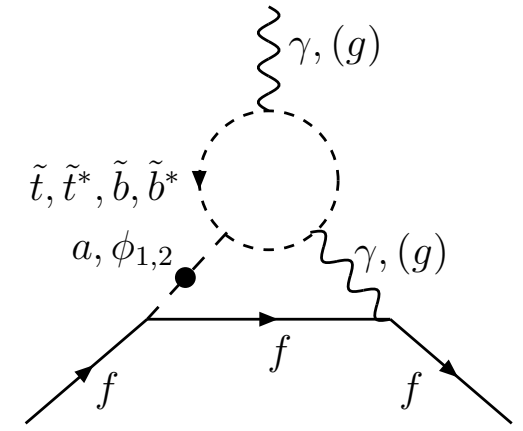

(a)

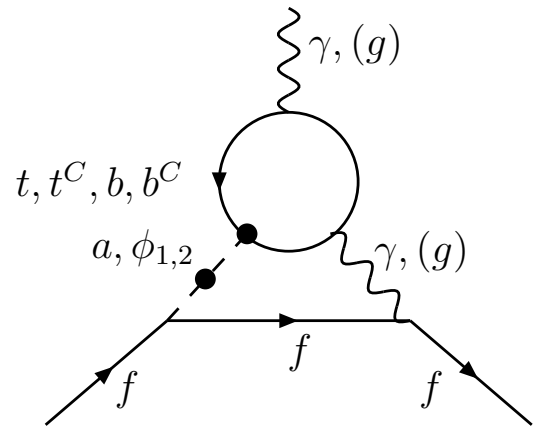

(c)

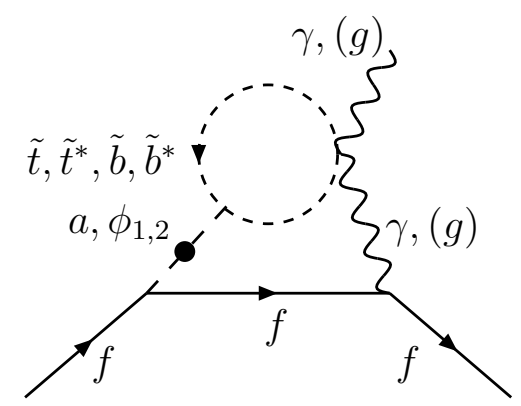

(b)

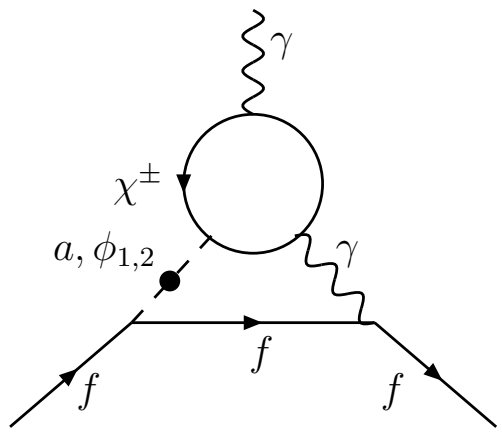

(d)

Figure 2: Dominant Higgs-boson two-loop contributions to EDM of a light fermion $f=$ $e, \mu, d$ in the MSSM with explicit CP violation (mirror-symmetric graphs are not displayed). Heavy dots indicate resummation of self-energy and vertex graphs. Two-loop graphs generating a CEDM for a d-quark are also shown. 
In the context of the aforementioned resummation approach, the dominant Higgsboson two-loop contributions to electron EDM are individually found to be

$$
\begin{aligned}
\left(\frac{d_{e}}{e}\right)_{(\mathrm{a}, \mathrm{b})}= & \frac{3 \alpha_{\mathrm{em}}}{32 \pi^{3}} m_{e} \sum_{i=1}^{3} \frac{g_{H_{i} e e}^{P}}{M_{H_{i}}^{2}} \sum_{q=t, b} Q_{q}^{2}\left\{\xi_{q}^{\left(H_{i}\right)}\left[F\left(\frac{m_{\tilde{q}_{1}}^{2}}{M_{H_{i}}^{2}}\right)-F\left(\frac{m_{\tilde{q}_{2}}^{2}}{M_{H_{i}}^{2}}\right)\right]\right. \\
& \left.+\zeta_{q}^{\left(H_{i}\right)}\left[F\left(\frac{m_{\tilde{q}_{1}}^{2}}{M_{H_{i}}^{2}}\right)+F\left(\frac{m_{\tilde{q}_{2}}^{2}}{M_{H_{i}}^{2}}\right)\right]\right\}, \\
\left(\frac{d_{e}}{e}\right)_{(\mathrm{c})}= & -\frac{3 \alpha_{\mathrm{em}}^{2}}{8 \pi^{2} \sin ^{2} \theta_{w}} \frac{m_{e}}{M_{W}^{2}} \\
& \times \sum_{i=1}^{3} \sum_{q=t, b} Q_{q}^{2}\left[g_{H_{i} e e}^{P} g_{H_{i} q q}^{S} f\left(\frac{m_{q}^{2}}{M_{H_{i}}^{2}}\right)+g_{H_{i} e e}^{S} g_{H_{i} q q}^{P} g\left(\frac{m_{q}^{2}}{M_{H_{i}}^{2}}\right)\right], \\
\left(\frac{d_{e}}{e}\right)_{(\mathrm{d})}= & -\frac{\alpha_{\mathrm{em}}^{2}}{8 \sqrt{2} \pi^{2} \sin ^{2} \theta_{w}} \frac{m_{e}}{M_{W}} \\
& \times \sum_{i=1}^{3} \sum_{j=1,2} \frac{1}{m_{\chi_{j}^{+}}}\left[g_{H_{i} e e}^{P} a_{H_{i} \chi_{j}^{-} \chi_{j}^{+}} f\left(\frac{m_{\chi_{j}^{+}}^{2}}{M_{H_{i}}^{2}}\right)+g_{H_{i} e e}^{S} b_{H_{i} \chi_{j}^{-} \chi_{j}^{+}} g\left(\frac{m_{\chi_{j}^{+}}^{2}}{M_{H_{i}}^{2}}\right)\right],
\end{aligned}
$$

where $g_{H_{i} e e}^{P}=-\tan \beta O_{3 i}, g_{H_{i} e e}^{S}=O_{1 i} / \cos \beta$, and

$$
\begin{aligned}
F(z) & =\int_{0}^{1} d x \frac{x(1-x)}{z-x(1-x)} \ln \left[\frac{x(1-x)}{z}\right] \\
f(z) & =\frac{z}{2} \int_{0}^{1} d x \frac{1-2 x(1-x)}{x(1-x)-z} \ln \left[\frac{x(1-x)}{z}\right] \\
g(z) & =\frac{z}{2} \int_{0}^{1} d x \frac{1}{x(1-x)-z} \ln \left[\frac{x(1-x)}{z}\right]
\end{aligned}
$$

are two-loop functions. The coupling coefficients $g_{H_{i} q q}^{S}, g_{H_{i} q q}^{P}, a_{H_{i} \chi_{j}^{-} \chi_{j}^{+}}$and $b_{H_{i} \chi_{j}^{-} \chi_{j}^{+}}$in (3.1)(3.3), as well as the squark and chargino masses, are given in the appendix. Equation (3.1) takes on the simpler analytic form presented in [2], if only the CP-odd component $a$ of the Higgs bosons is considered in an unresummed two-loop calculation of the EDM. In this case, the coefficients $\zeta^{\left(H_{i}\right)}$ vanish and $\xi_{q}^{\left(H_{i}\right)}$ simplifies to

$$
\xi_{q}=R_{q} \frac{\sin 2 \theta_{q} m_{q} \operatorname{Im}\left(\mu e^{i \delta_{q}}\right)}{\sin \beta \cos \beta v^{2}}=\frac{R_{q}}{\sin \beta \cos \beta} \frac{2 m_{q}^{2} \operatorname{Im}\left(\mu A_{q}\right)}{v^{2}\left(m_{\tilde{q}_{2}}^{2}-m_{\tilde{q}_{1}}^{2}\right)},
$$

where $\delta_{q}=\arg \left(A_{q}-R_{q} \mu^{*}\right)$, with $R_{t}\left(R_{b}\right)=\cot \beta(\tan \beta)$.

In addition to the dominant Higgs-boson two-loop graphs we have been studying here, there are also subdominant two-loop EDM diagrams, where the virtual photon is replaced by a $Z$ boson in Fig. 2. Another class of Higgs-boson two-loop graphs involve the coupling of the charged Higgs bosons $H^{ \pm}$to the photon and the $W^{\mp}$ bosons [3]. In this case, for 
example, the graph in Fig. 2 will proceed via charginos and neutralino in the fermionic loop. As has been explicitly shown in [3] for most of the cases, this additional set of graphs give almost one order of magnitude smaller contributions to EDM. Most importantly, their dependence on the CP-violating parameters of the theory is rather closely related to the two-loop EDM graphs depicted in Fig. 2. Thus, suppressing the dominant Higgs-boson twoloop contributions to EDM will automatically lead to a corresponding suppression of this additional set of two-loop graphs. Therefore, in our analysis we neglect the aforementioned set of subdominant two-loop graphs.

So far, we have only been studying the electron EDM $d_{e}$. The two-loop prediction for the muon EDM $d_{\mu}$ can easily be obtained from $d_{e}$ by considering the obvious mass rescaling factor $m_{\mu} / m_{e} \approx 205$, i.e.

$$
d_{\mu} \approx 205 d_{e}\left(t, b, \tilde{t}, \tilde{b}, \chi^{ \pm}\right)
$$

where the different two-loop EDM contributions are indicated within the parentheses.

On the other hand, the dominant contributions to neutron EDM $d_{n}$ come from the CEDM of the $d$ quark and CP-odd three-gluon operator [30], which was first discussed by Weinberg [29] in non-supersymmetric multi-Higgs doublet models. In the MSSM, the CP-odd three-gluon operator decouples as $1 / m_{\tilde{g}}^{3}$ and becomes relevant at gluino masses below the TeV scale. The CEDM of the $d$ quark may be obtained from (3.1) and (3.2), if one replaces the colour factor 3 by $1 / 2$, and $\alpha_{\mathrm{em}} Q_{q}^{2}$ by $\alpha_{s}$. The computation of the neutron $\mathrm{EDM} d_{n}$ involves a number of hadronic uncertainties, when the EDMs are translated from the quark to the hadron level [25]. For example, considering the valence-quark model and renormalization-group running effects from the electroweak scale $M_{Z}$ down to the lowenergy hadronic scale $\Lambda_{h}$ [3], one may be able to establish an approximate relation between neutron and electron EDMs. Thus, taking the input values for the involved kinematic parameters: $m_{d}\left(\Lambda_{h}\right)=10 \mathrm{MeV}, \alpha_{s}\left(M_{Z}\right)=0.12$ and $g_{s}\left(\Lambda_{h}\right) /(4 \pi)=1 / \sqrt{6}$, we find

$$
d_{n} \approx-10 d_{e}(t, \tilde{t})+1.2 d_{e}\left(t, \tilde{t}, \chi^{ \pm}\right)+d_{n}^{3 G}
$$

On the RHS of (3.9), the first and second terms arise from a $d$-quark CEDM and EDM, respectively, and $d_{n}^{3 G}$ is the contribution to $d_{n}$ due to the $\mathrm{CP}$-odd three-gluon operator. In obtaining (3.9), we have made two additional approximations as well. First, we neglected the contribution of the $u$-quark $\operatorname{EDM} d_{u}$ to $d_{n}$, as it is much smaller than the $d$-quark EDM $d_{d}$ for the relevant region $\tan \beta \gtrsim 3$. Second, we ignored the $b$ - and $\tilde{b}$-quantum corrections to $d_{d}$ and so to $d_{n}$. Formulae (3.8) and (3.9) will be used to obtain numerical predictions for the muon and neutron EDMs in the next section. 


\section{Numerical estimates and discussion}

In Sections 2 and 3, we computed the dominant two-loop and the resummed higherloop contributions to EDMs that originate from third-generation quarks and squarks, and charginos. Based on the derived analytic expressions, we can now analyze numerically the impact of the experimental constraints due to the non-observation of thallium and neutron EDMs on the CP-violating parameters of the theory, and hence on electroweak baryogenesis and direct searches for CP-violating Higgs bosons in the MSSM. Moreover, we will present predictions for the muon EDM $d_{\mu}$ and discuss the implications of a possible high-sensitivity measurement of $d_{\mu}$ to the level $10^{-24} e \mathrm{~cm}$ for our analyses.

Based on the observation that CP-violating quantum effects on the neutral Higgs sector get enhanced when the product $\operatorname{Im}\left(\mu A_{t}\right) / M_{\text {SUSY }}^{2}$ is large [5, 6], the authors in [8, 9] introduced a benchmark scenario, called CPX, in which the effects of CP violation are maximized. In CPX, the following values for the $\mu$ - and soft-SUSY-breaking parameters were adopted:

$$
\begin{aligned}
\widetilde{M}_{Q} & =\widetilde{M}_{t}=\widetilde{M}_{b}=M_{\mathrm{SUSY}}, \quad \mu=4 M_{\mathrm{SUSY}} \\
\left|A_{t}\right| & =\left|A_{b}\right|=2 M_{\mathrm{SUSY}}, \quad \arg \left(A_{t, b}\right)=90^{\circ} \\
\left|m_{\tilde{g}}\right| & =1 \mathrm{TeV}, \quad \arg \left(m_{\tilde{g}}\right)=90^{\circ}, \\
m_{\widetilde{W}} & =m_{\widetilde{B}}=0.3 \mathrm{TeV} .
\end{aligned}
$$

Without loss of generality, the $\mu$-parameter is chosen to be real. The predictions of CPX showed [9] that even a light neutral Higgs boson with a mass as low as $60 \mathrm{GeV}$ could have escaped detection at LEP2. A recent experimental analysis of LEP2 data confirms this observation [43]. Here, we wish to investigate the compatibility of the CPX scenario with the experimental limits on EDMs. For this purpose, we allow variations in the gluino phase, which enters the Higgs sector at two loops, but keep the $A_{t}$ phase in (4.1) fixed. In addition, we will present numerical results for EDMs, where the $\mu$-parameter is varied from $100 \mathrm{GeV}$ to $4 M_{\mathrm{SUSY}}$. Finally, we leave unspecified the phases of the gaugino mass parameters $m_{\widetilde{W}}$ and $m_{\widetilde{B}}$. As we will see below, the phase of $m_{\widetilde{W}}$ is greatly affected by constraints from the electron EDM.

We start our numerical analysis by presenting predicted values for the ${ }^{205} \mathrm{Tl}$ EDM $d_{\mathrm{Tl}}$ that arise entirely due to the CP-odd electron-nucleon operator $C_{S}$ and are denoted as $d_{\mathrm{Tl}}\left(C_{S}\right)$. In Fig. 3, we display numerical estimates for $d_{\mathrm{Tl}}\left(C_{S}\right)$ as functions of $\tan \beta$ for four different versions of the CPX scenario with $M_{\mathrm{SUSY}}=1 \mathrm{TeV}$ : (a) $M_{H^{+}}=150 \mathrm{GeV}$,

\footnotetext{
${ }^{3}$ Similar remarks were made earlier in [6], but the LEP2 data were less restrictive then.
} 
$\arg \left(m_{\tilde{g}}\right)=0^{\circ} ;(\mathrm{b}) M_{H^{+}}=300 \mathrm{GeV}, \arg \left(m_{\tilde{g}}\right)=0^{\circ} ;$ (c) $M_{H^{+}}=150 \mathrm{GeV}, \arg \left(m_{\tilde{g}}\right)=90^{\circ}$; (d) $M_{H^{+}}=300 \mathrm{GeV}$, $\arg \left(m_{\tilde{g}}\right)=90^{\circ}$. The individual $b, \tilde{t}, t, \tilde{b}$ contributions to $d_{\mathrm{Tl}}\left(C_{S}\right)$, along with their relative signs, are indicated by different types of lines. We observe that the largest contribution to $d_{\mathrm{Tl}}$ comes from the $b$-quarks for large values of $\tan \beta$, i.e. for $\tan \beta \gtrsim 15$, for which the CP-violating vertex effects become important (see also the discussion in Section 2). In particular, these CP-violating threshold effects, which crucially depend on the term $\operatorname{Im}\left(\Delta h_{b} / h_{b}\right) \tan ^{2} \beta$ in (2.3), become even more important for large gluino phases. Thus, the predictions for $d_{\mathrm{Tl}}\left(C_{S}\right)$ in panels $3(\mathrm{a})$ and $(\mathrm{b})$, with $\arg \left(m_{\tilde{g}}\right)=0^{\circ}$, are one order of magnitude larger than the ones in (c) and (d), with $\arg \left(m_{\tilde{g}}\right)=90^{\circ}$.

For intermediate and smaller values of $\tan \beta$, i.e. for $\tan \beta \lesssim 15$, CP-violating selfenergy effects are significant, especially for relatively light charged Higgs bosons with masses in the range 150-200 GeV. In fact, these effects have generically opposite sign to the $\mathrm{CP}$-violating vertex effects, giving rise to natural cancellations among the contributing EDM terms, and so lead to smaller values of $d_{\mathrm{Tl}}\left(C_{S}\right)$. Although our numerical results are in qualitative agreement with those in Ref. [40], we actually find noticeable quantitative differences, when resummed CP-violating self-energy and vertex effects are considered at the same time.

Next, we shall investigate numerically higher-order CP-violating vertex and selfenergy effects induced by $t$ - and $b$-quarks on the electron EDM $d_{e}$. Fig. 1 shows numerical estimates for those resummed effects on $d_{e}$ as functions of $\tan \beta$, in variants of the CPX scenario, with (a) $M_{H^{+}}=150 \mathrm{GeV}$ and (b) $M_{H^{+}}=300 \mathrm{GeV}$. In particular, we considered three different choices of the gluino phase: $\arg \left(m_{\tilde{g}}\right)=90^{\circ}, 0,-90^{\circ}$, denoted as $t_{1,2,3}$, respectively. We find that CP-violating threshold corrections to the $H_{i} t t$ coupling as small as $5 \%$ are sufficient to lead to observable EDM values for $d_{e}$. In this respect, we see that the $t$-quark effects strongly depend on the gluino phase through the combination $\operatorname{Im}\left(\mu m_{\tilde{g}}\right)$ that occurs in $\operatorname{Im}\left(\Delta h_{t} / h_{t}\right)$ [cf. (A.4), (A.5)]. Thus, the $t$-quark contribution to $d_{e}$ is positive (negative) for negative (positive) gluino phases, while it is one order of magnitude smaller and negative for vanishing gluino phases, i.e. for $\arg \left(m_{\tilde{g}}\right)=0^{\circ}$. For comparison, we also included in Fig. \& the dependence of positive stop/sbottom contributions to $d_{e}$ [2] (long-dash-dotted lines) on $\tan \beta$. The sum of the $t$-, $b$-quark and $\tilde{t}$-, $\tilde{b}$-squark contributions to $d_{e}$ is given by the solid lines 1,2,3 for the same values of gluino phases. As before, we indicate negative contributions to $d_{e}$ with a minus sign. From Figs. 4 (a) and (b), it is interesting to notice that if $\arg \left(m_{\tilde{g}}\right)=90^{\circ}$ in CPX, a cancellation between the $t$-quark and $\tilde{t}$-squark EDM contributions occurs for almost the entire range of the perturbatively allowed $\tan \beta$ values and for all phenomenologically viable charged Higgs-boson masses. 
As a consequence of such a cancellation, the electron EDM $d_{e}$ is always smaller than the current $2 \sigma$ experimental limit on $d_{e}$, i.e. $d_{e}<2.2 \times 10^{-27} e \mathrm{~cm}$, even for large tan $\beta$ values up to 30. As we will see below, this prediction may considerably change if contributions from the CP-violating operator $C_{S}$ or chargino two-loop effects are considered.

In order to further gauge the importance of the $t$-quark two-loop EDM effects, we present in Fig. 5 numerical values for $d_{e}$ versus the $\mu$-parameter for $\tan \beta=20$, and for two charged Higgs-boson masses: (a) $M_{H^{+}}=150 \mathrm{GeV}$ and (b) $M_{H^{+}}=300 \mathrm{GeV}$. The softSUSY-breaking parameters are chosen as given in (4.1) for $M_{\mathrm{SUSY}}=1 \mathrm{TeV}$, except for the $\mu$-parameter, which has been varied from 0.1-4 TeV. For the sake of comparison, we also included the Higgs-boson two-loop EDM effects induced by $\tilde{t}$ - and $\tilde{b}$-squarks. The meaning of the various types of lines is exactly the same as those in Fig. 4. Remarkably enough, we find that even $\mu$ values as low as $500 \mathrm{GeV}$ may be sufficient to lead to an electron EDM at the observable level through the original two-loop Barr-Zee graph in Fig. 2(c). In this context, we also observe that the resummed Higgs-boson two-loop contributions to $d_{e}$ from $t$-quarks are comparable and even larger than those coming from $\tilde{t}$-squarks for maximal gluino phases. In fact, if $A_{t, b}=0$, the $\tilde{t}$-squark and dominant CP-violating Higgs-mixing effects may be completely switched off, without much affecting the corresponding $t$-quark two-loop contributions to $d_{e}$. Note that in this case the $t$-quark effects on $d_{e}$ and the $b$-quark effects on the $C_{S}$ operator, which both formally arise at the two-loop level, are proportional to $\operatorname{Im}\left(\mu m_{\tilde{g}}\right)$. Therefore, they turn out to be strongly correlated and their combined contribution to $d_{\mathrm{Tl}}$ should carefully be taken into account (see also discussions of Figs. 7 and 8 below).

As was already pointed out in [2, 3], charginos might also contribute to electron EDM $d_{e}$ at the two-loop level. Recently, a computation of those effects appeared in [41. The authors derived strict constraints on the CP-violating parameters of a scenario in which electroweak baryogenesis is mediated by CP-violating currents involving chargino interactions. Here, we re-examine this issue within a scenario that favours the above mechanism of electroweak baryogenesis and is not in conflict with LEP2 limits on the Higgs-boson masses and couplings. Specifically, being conservative, we require that these be $M_{H_{i}} \gtrsim 111 \mathrm{GeV}$, for $g_{H_{i} Z Z}^{2} \gtrsim 0.3$, where $g_{H_{i} Z Z}$ is the $H_{i} Z Z$ coupling given in units of the SM $h_{\mathrm{SM}} Z Z$ coupling. In addition, we demand that $M_{H_{i}}+M_{H_{j}} \gtrsim 170 \mathrm{GeV}$. On the other hand, in order for electroweak baryogenesis to proceed via a sufficiently strong firstorder phase transition, the right-handed stop mass parameter $\widetilde{M}_{t}$ must be rather small, and the $\mu$ and the soft gaugino parameter $m_{\widetilde{W}}$ must not be too large, typically smaller than $0.5 \mathrm{TeV}$ [13, 14]. Especially, there is a resonant enhancement up to even 10 times the 
observed baryon asymmetry, if the condition $\mu=m_{\widetilde{W}}$ is met [14. Further requirements for a scenario leading to successful electroweak baryogenesis are: (i) a moderate trilinear $A_{t}$-parameter in the range, $0.2 \lesssim A_{t} / \widetilde{M}_{Q} \lesssim 0.65$; (ii) a not very large $\tan \beta$ value, $\tan \beta \lesssim 20$; (iii) a soft-SUSY-breaking parameter $\widetilde{M}_{Q}$ of a few $\mathrm{TeV}$, for phenomenological reasons [14. More explicitly, the following values for the mass parameters are employed:

$$
\begin{aligned}
\widetilde{M}_{Q} & =3 \mathrm{TeV}, \quad \widetilde{M}_{t}=0, \quad \widetilde{M}_{b}=3 \mathrm{TeV}, \\
\left|A_{t}\right| & =\left|A_{b}\right|=1.8 \mathrm{TeV}, \quad \arg \left(A_{t, b}\right)=0^{\circ}, \quad \tan \beta \lesssim 20, \\
\left|m_{\tilde{g}}\right| & =3 \mathrm{TeV}, \quad \arg \left(m_{\tilde{g}}\right)=0^{\circ}, \\
\mu & =\left|m_{\widetilde{W}}\right| \lesssim 0.5 \mathrm{TeV}, \quad \arg \left(m_{\widetilde{W}}\right)=90^{\circ} .
\end{aligned}
$$

To be able to compare our predictions with those presented in Fig. 2 of Ref. 41, we choose in Fig. 6(a) the values: $\mu=m_{\widetilde{W}}=0.2 \mathrm{TeV}$ and $M_{H^{+}}=170 \mathrm{GeV}$. Since CP-violating Higgs-mixing effects in the mass spectrum are generically small for the chosen values of the parameters in (4.2), our mass input $M_{H^{+}}=170 \mathrm{GeV}$ corresponds to $M^{\prime} A^{\prime} \approx 150 \mathrm{GeV}$ for the mass of the almost CP-odd Higgs scalar $A$. Even though on a very qualitative basis our numerical results on the linear $\tan \beta$-increase behaviour of $d_{e}$ agree with those reported in [41], the actual functional dependences of the individual ' $h$ ', ' $H$ ', ' $A$ ' contributions to $d_{e}$ on $\tan \beta$ differ significantly. Unlike [41], we find in Fig. 6(a) that for $\tan \beta \gtrsim 5$, the $\tan \beta$-enhanced effect on $d_{e}$ originates from the heavier Higgs bosons ' $H$ ' and ' $A$ ', while the EDM contribution due to the lightest Higgs boson ' $h$ ' is almost negligible. $f$ Since the size of $d_{e}$ is set by the heavier Higgs-boson masses, i.e. by $M_{H^{+}}$, and by $\mu$ and $m_{\widetilde{W}}$, our predictions are rather robust under the different choices of the remaining soft-SUSYbreaking parameters. Moreover, although our numerical values for the total contribution to $d_{e}$ agree very well with 441 for $\tan \beta=2\left(d_{e} \approx 0.63 \times 10^{-26} e \mathrm{~cm}\right)$, they are smaller by $\sim 20 \%$ for $\tan \beta=6$, i.e. we find $d_{e} \approx 1.62 \times 10^{-26} e \mathrm{~cm}$, which should be compared with $d_{e} \approx 2 \times 10^{-26} e \mathrm{~cm}$. Finally, the electroweak baryogenesis scenario (4.2) in the low $\tan \beta$ region, $\tan \beta \lesssim 6$, which is studied by the authors in [41], appears to be highly disfavoured by LEP2 data. In this respect, a phenomenologically viable model, with $M_{H^{+}}=170 \mathrm{GeV}$, would require larger values of $\tan \beta$, i.e. $\tan \beta \approx 9$. In this case, one has to consider a factor of 10 suppression in the chargino phase, such that the chargino two-loop EDM effects are reduced to a level close to the experimental upper limit on $d_{e}$. Consequently, if no cancellations are assumed with possible one-loop EDM terms, then a model with suppressed chargino phase of $\sim 5^{\circ}$ and a relatively light charged Higgs boson, $M_{H^{+}}=150-200 \mathrm{GeV}$,

\footnotetext{
${ }^{4}$ The fact that only ' $H$ ' and ' $A$ ' contributions to $d_{e}$ exhibit a linearly enhanced dependence on tan $\beta$ may also be verified independently by a flavour-graph analysis.
} 
might still be possible to account for the observed baryon asymmetry in the Universe, provided the aforementioned resonant factor 10 is used. However, the above situation may be considerably relaxed for larger values of $M_{H^{+}}$, since the chargino two-loop EDM effect on $d_{e}$ decreases approximately by $1 / M_{H^{+}}$as $M_{H^{+}}$increases. This dependence of $d_{e}$ on $M_{H^{+}}$can explicitly be seen in the lower panel (b) of Fig. 6, for increasing charged Higgs-boson masses: $M_{H^{+}}=150 \mathrm{GeV}$ (solid), $200 \mathrm{GeV}$ (dashed), $300 \mathrm{GeV}$ (dotted), $500 \mathrm{GeV}$ (dash-dotted) and $1 \mathrm{TeV}$ (long-dash-dotted), in a scenario with $m_{\widetilde{W}}=\mu=0.4 \mathrm{TeV}$ and $\arg \left(m_{\widetilde{W}}\right)=90^{\circ}$.

In the following, we will present predictions for more realistic EDM observables, with relatively reduced hadronic uncertainties, namely the thallium EDM $d_{\mathrm{Tl}}$, the neutron EDM $d_{n}$, as well as the muon EDM $d_{\mu}$ which was suggested to be measured with a high sensitivity to the level of $10^{-24} e \mathrm{~cm}$ [20]. In Fig. 7, we display numerical values for $d_{\mathrm{Tl}}, d_{n}$ and $d_{\mu}$ as functions of $\tan \beta$ in two versions of the CPX scenario, with $M_{H^{+}}=150 \mathrm{GeV}$ : (a) $\arg \left(m_{\tilde{g}}\right)=\arg \left(m_{\widetilde{W}}\right)=90^{\circ}$, and $(\mathrm{b}) \arg \left(m_{\tilde{g}}\right)=35^{\circ}, \arg \left(m_{\widetilde{W}}\right)=90^{\circ}$. Fig. 7 also shows the different contributions, along with their relative signs, to $d_{\mathrm{Tl}}$ from top/stop (long-dashdotted) and chargino (dotted) Higgs-boson two-loop graphs, as well as from the CP-odd electron-nucleon operator $C_{S}$ (dashed). Note that the type of lines used to represent the numerical results of the individual EDM contributions is given in the parentheses. In panel (a) of Fig. 7, we see that the contribution of $C_{S}$ prevails in $d_{\mathrm{Tl}}$, for large values of $\tan \beta$, and exceeds the experimental limit for $\tan \beta \gtrsim 12$. The prediction for $d_{n}$ always stays below the current experimental limit, and the predicted values for $d_{\mu}$ do not reach the proposed experimental sensitivity for almost all relevant values of $\tan \beta$. It is amusing to remark that no EDM constraints can be imposed on the CPX scenario in the range: $4 \lesssim \tan \beta \lesssim 12$, which is interesting for analyzing Higgs-boson searches at high-energy colliders. In fact, if the gluino phase is chosen to be $\arg \left(m_{\tilde{g}}\right)=35^{\circ}$ (see Fig. 7(b)), the different EDM terms contributing to $d_{\mathrm{Tl}}$ approximately cancel and $d_{\mathrm{Tl}}$ does not exceed much the experimental limit. Similarly, since the top/stop- CEDM effects are small in this CPX scenario, the neutron EDM is always smaller than its conservative experimental upper bound: $1.2 \times 10^{-25} \mathrm{e} \mathrm{cm}$. However, for $\tan \beta \approx 40$, the muon EDM can be significant, and its value $d_{\mu} \sim 4 . \times 10^{-24} e \mathrm{~cm}$ lies well within the proposed explorable range. This example nicely illustrates the important rôle of complementarity of a high-sensitivity measurement of a muon EDM in constraining the CP-violating parameter space of the MSSM.

It is also interesting to examine the dependence of the different EDM contributions shown in Fig. 7 on the $\mu$-parameter, for large values of $\tan \beta$. In Fig. 8, we display numerical values of $d_{\mathrm{Tl}}, d_{n}$ and $d_{\mu}$ as functions of $\mu$ for two scenarios with $\tan \beta=40, M_{\mathrm{SUSY}}=1 \mathrm{TeV}$, $m_{\tilde{g}}=1 \mathrm{TeV}, m_{\widetilde{W}}=m_{\widetilde{B}}=0.3 \mathrm{TeV}, \arg \left(m_{\tilde{g}}\right)=\arg \left(m_{\widetilde{W}}\right)=90^{\circ}, A_{t, b}=2 \mathrm{TeV}, \arg \left(A_{t, b}\right)=$ 
90: (a) $M_{H^{+}}=150 \mathrm{GeV}$; (b) $M_{H^{+}}=300 \mathrm{GeV}$. In analogy with Fig. 7, the individual contributions to $d_{\mathrm{Tl}}$ due to top/stop and chargino two-loop graphs and due to the CP-odd electron-nucleon operator $C_{S}$ are also shown. In Fig. 8(a), we observe that the different CP-violating EDM operators may cancel in $d_{\mathrm{Tl}}$ and $d_{n}$, even for smaller values of the $\mu$ parameter, i.e. for $\mu \approx 700 \mathrm{GeV}$. In this region of parameter space, the muon EDM is predicted to be as large as $0.8 \times 10^{-23} \mathrm{e} \mathrm{cm}$, which falls within the reach of the proposed $d_{\mu}$ measurement. In Fig. 8(b), we give numerical estimates of $d_{\mathrm{Tl}}, d_{n}$ and $d_{\mu}$ for a CPX scenario with a heavier charged Higgs boson, i.e. for $M_{H^{+}}=300 \mathrm{GeV}$. Again, we find that the predicted value for $d_{\mathrm{Tl}}$ can be close to the experimental limit for a wide range of $\mu$-values, while $d_{\mu}$ always stays above the proposed experimental sensitivity.

Let us summarize the focal points of this section. We have explicitly demonstrated that the non-observation of the thallium EDM can provide strict constraints on the CPviolating parameters related to third-generation squarks, charginos and gluinos. The constraints derived from the neutron EDM limit are less restrictive. Nevertheless, our numerical analysis has also shown that the constraints from the thallium EDM can be significantly weakened, if the different CP-violating operators $d_{e}$ and $C_{S}$ cancel in $d_{\mathrm{Tl}}$. For instance, this could be the case for the benchmark scenario CPX, for low and intermediate values of $\tan \beta$. Such cancellations of the CP-violating operators $d_{e}$ and $C_{S}$ can occur for a wide range of parameters and crucially depend on the choice of the phase combinations: $\arg \left(\mu A_{t}\right)$, $\arg \left(\mu m_{\tilde{g}}\right)$ and $\arg \left(\mu m_{\tilde{W}}\right)$. In particular, we find that a possible high-sensitivity measurement of $d_{\mu}$ to the proposed level of $10^{-24} e \mathrm{~cm}$ can constrain such uncovered ranges of CP-violating parameters in a rather complementary way. Finally, unless $M_{H^{+}}$is of the TeV order, EDM constraints from $d_{\mathrm{Tl}}$ on scenarios favoured by electroweak baryogenesis are rather stringent. They generally imply either suppressed chargino phases, i.e. $\arg \left(m_{\widetilde{W}}\right) \lesssim 10^{\circ}$, or modest cancellations in 1 part to 10 with one-loop EDM terms induced by the first two generations of sleptons.

\section{Conclusions}

To avoid the known CP and FCNC crises in the MSSM, we have considered a framework, in which the first two generations of squarks and sleptons are heavier than $\sim 10 \mathrm{TeV}$, while the third generation is light, with masses not larger than the order of a TeV. Within this framework of the MSSM, we have performed a systematic study of the dominant two- and higher-loop contributions to the thallium, neutron and muon EDMs, which are induced by

$b$-, $t$-quarks, $\tilde{b}$-, $\tilde{t}$-squarks, charginos and gluinos. At present, the most severe limits are 
obtained from the non-observation of a thallium EDM $d_{\mathrm{Tl}}$, whereas experimental upper limits on the neutron EDM $d_{n}$ are less stringent and usually constrain large contributions from a $d$-quark CEDM and the CP-odd three-gluon operator. Also, theoretical predictions for $d_{n}$ are plagued by a number of uncertainties while estimating hadronic matrix elements.

The largest effects on the thallium EDM $d_{\mathrm{Tl}}$ result from two operators, the CP-odd electron-nucleon operator $C_{S}$ and the electron EDM $d_{e}$. These two CP-violating operators are formally induced at the two- and higher-loop levels and involve the exchange of CPmixed Higgs bosons. Thus, strong constraints on the radiatively-generated CP-violating Higgs sector of the MSSM can be derived from $d_{\mathrm{Tl}}$, and hence on the analyses for direct searches of CP-violating Higgs bosons at high-energy colliders, such as LEP2, Tevatron and LHC [44]. In this context, we have analyzed the compatibility of an earlier suggested benchmark scenario of maximal CP violation for LEP2 Higgs studies (CPX) [9] with the thallium and neutron EDMs. We have observed the existence of strong correlations among the different EDM terms, which enable the suppression of $d_{\mathrm{Tl}}$ and $d_{n}$ even below the present experimental limits. Specifically, for $4 \lesssim \tan \beta \lesssim 12$ in the CPX scenario with $M_{H^{+}}=150 \mathrm{GeV}$, the stop, gluino, and chargino phases are all allowed to receive their maximal values, i.e. $\arg \left(A_{t}\right)=\arg \left(m_{\tilde{g}}\right)=\arg \left(m_{\widetilde{W}}\right)=90^{\circ}$, without being in conflict with EDM limits (cf. Fig. [1(a)). Most interestingly, for specific choices of the gluino phase, the allowed range of $\tan \beta$ values compatible with EDM limits can be enlarged dramatically. For instance, if $\arg \left(m_{\tilde{g}}\right)=35^{\circ}$ in the aforementioned CPX scenario (see also Fig. $7(\mathrm{~b})$ ), the predicted values for $25 \lesssim \tan \beta \lesssim 45$ do not contradict upper limits on thallium and neutron EDMs. For the remaining range of $\tan \beta$ values, the obtained prediction does not exceed the $2 \sigma$ upper bound on $\left|d_{\mathrm{Tl}}\right|$ by a factor of $\sim 3$. Evidently, the degree of cancellations required between the one- and two-loop EDM terms in the CPX scenario is not excessive, for certain choices of the gluino phase.

At this point, it is important to stress that a muon EDM $d_{\mu}$ measured at the $10^{-24}$ e cm-level will help to sensitively probe CP-violating regions of the MSSM parameter space which cannot be accessed easily by measurements of the thallium and neutron EDMs. This complementarity property is mainly a consequence of the fact that $d_{\mu}$ is free from interfering $\mathrm{CP}$-odd electron-nucleus interactions thanks to the $C_{S}$ operator, which can contribute significantly to $d_{\mathrm{Tl}}$. Unlike the neutron EDM $d_{n}, d_{\mu}$ does not suffer from hadronic uncertainties. Given the absence of a signal in the measurements of $\left|d_{\mathrm{Tl}}\right|$ and $\left|d_{n}\right|$, one may now wonder whether a positive signal in $d_{\mu}$ would already imply a positive signal on $g-2$ as well. This is not the case within our framework of the MSSM. If the first two generations of sfermions are above the $\mathrm{TeV}$ scale, the biggest contribution to $g-2$ comes 
again from related two-loop Barr-Zee-type graphs. However, for phenomenologically viable charged Higgs-boson masses $M_{H^{+}} \gtrsim 120 \mathrm{GeV}$ in the MSSM [43], these effects on $g-2$ are negligible [45]. Then, only post-LEP2 high-energy colliders and the proposed BNL experiment 20] on the muon EDM $d_{\mu}$ might be able to sensitively explore the CP-violating parameter space of the above framework of the MSSM in a rather complementary manner.

We have also studied the impact of EDM constraints on the mechanism of electroweak baryogenesis induced by CP-violating chargino currents. For this purpose, we considered a scenario in (4.2), which favours the above mechanism of electroweak baryogenesis [14]. In such a scenario, the chargino two-loop graphs of Fig. (2)(d) represent the dominant contribution to $d_{e}$ and $d_{\mathrm{Tl}}$ as well. However, as we detailed in Section 4, our theoretical predictions for $d_{e}$ are at variance with those presented in a recent communication [41]. Moreover, we find that LEP2 direct limits on Higgs-boson masses require intermediate and larger values of $\tan \beta$, i.e. $\tan \beta \gtrsim 6$, for a phenomenologically viable scenario of electroweak baryogenesis. In this $\tan \beta$ regime, experimental upper limits on $\left|d_{\mathrm{Tl}}\right|$ give rise to strict constraints, especially when no cancellations between the chargino two-loop and one-loop EDM terms are assumed. In the latter case, the charged Higgs-boson mass $M_{H^{+}}$should be relatively large, i.e. $M_{H^{+}} \gtrsim 700 \mathrm{GeV}$ for $\tan \beta \gtrsim 6$ and $\arg \left(m_{\widetilde{W}}\right) \lesssim 90^{\circ}$. Otherwise, for lighter charged Higgs bosons, either the chargino phase should be suppressed by a factor of at least 10 or cancellations in 1 part to 10 with one-loop EDM terms need be invoked.

In our computation of the Higgs-boson loop-induced EDMs, we have considered resummation effects of higher-order CP-conserving and CP-violating terms in Higgs-boson self-energies and vertices. In particular, the original $t$-quark two-loop graph suggested by Barr and Zee [4] occurs beyond the two-loop approximation through threshold effects in the $H_{i} \bar{t} t$ coupling and, depending on the choice of the gluino phase, it might even compete with the $\tilde{t}$-squark two-loop graph [2]. Since our resummation of higher-order terms relied on an effective Lagrangian approach, one may worry about the relevance of other higherorder terms present in a complete computation. At this stage, we can only offer estimates of those possible higher-order electroweak uncertainties in the calculation of EDMs. Thus, we have checked our results with and without resumming the Higgs-boson self-energies. In this way, no large modifications are found in our predictions; the variation of our results is generally less than $10 \%$ for $M_{H^{+}} \lesssim 170 \mathrm{GeV}$, and becomes even smaller, to less than $1 \%$ for $M_{H^{+}} \gtrsim 200 \mathrm{GeV}$. This may be attributed to the fact that the dominant contributions to EDMs come from the heaviest Higgs bosons, on which the relative impact of radiative effects is less important. On the other hand, CP-violating threshold effects constitute the main source of theoretical uncertainties in the calculation of the original Barr-Zee graph 
of Fig. 2(c), as they are less controllable for low values of $\tan \beta$. In this context, we remark that even the computation of the CP-odd three-gluon operator is haunted by relevant higher-order electroweak uncertainties in the MSSM [30. The Weinberg operator can be generated in its original fashion [29] at three and higher loops which involve CP-violating self-energy and vertex subgraphs of Higgs bosons. It then appears necessary to develop improved techniques that would enable us to provide accurate estimates of (resummed) higher-order terms in the calculation of EDMs. The present work is a step towards this goal.

\section{Acknowledgements}

The author thanks Marcela Carena and Carlos Wagner for discussions on issues related to electroweak baryogenesis, Adam Ritz for comments on the computation of the mercury EDM, Maxim Pospelov for clarifying remarks pertinent to [40], Darwin Chang and Wai-Yee Keung for communications with regard to [41], and Athanasios Dedes for critical remarks.

\footnotetext{
${ }^{5} \mathrm{~A}$ crude estimate suggests that these additional higher-order effects are smaller than $20 \%$.
} 


\section{A Effective Higgs-boson couplings}

The couplings of the CP-mixed Higgs bosons $H_{1,2,3}$ to $t$-, $b$-quarks, $\tilde{t}$-, $\tilde{b}$-squarks and charginos $\chi^{+}$play a key rôle in our calculations. In this appendix we present the effective Lagrangians describing the above interactions, after including dominant one- and two-loop CP-even/CP-odd quantum effects on the Higgs-boson masses and their respective mixings.

Following the conventions of [8], we first write down the effective Lagrangian of the Higgs-boson couplings to top and bottom quarks

$$
\mathcal{L}_{H \bar{q} q}=-\sum_{i=1}^{3} H_{i}\left[\frac{g_{w} m_{b}}{2 M_{W}} \bar{b}\left(g_{H_{i} b b}^{S}+i g_{H_{i} b b}^{P} \gamma_{5}\right) b+\frac{g_{w} m_{t}}{2 M_{W}} \bar{t}\left(g_{H_{i} t t}^{S}+i g_{H_{i} t t}^{P} \gamma_{5}\right) t\right],
$$

with $8 \|^{0}$

$$
\begin{aligned}
& g_{H_{i} b b}^{S}=\operatorname{Re}\left[\frac{1+\left(\delta h_{b} / h_{b}\right)}{1+\left(\delta h_{b} / h_{b}\right)+\left(\Delta h_{b} / h_{b}\right) \tan \beta}\right] \frac{O_{1 i}}{\cos \beta} \\
& +\operatorname{Re}\left[\frac{\left(\Delta h_{b} / h_{b}\right)}{1+\left(\delta h_{b} / h_{b}\right)+\left(\Delta h_{b} / h_{b}\right) \tan \beta}\right] \frac{O_{2 i}}{\cos \beta} \\
& +\operatorname{Im}\left[\frac{\left(\Delta h_{b} / h_{b}\right)\left(\tan ^{2} \beta+1\right)}{1+\left(\delta h_{b} / h_{b}\right)+\left(\Delta h_{b} / h_{b}\right) \tan \beta}\right] O_{3 i}, \\
& g_{H_{i} b b}^{P}=-\operatorname{Re}\left\{\frac{\left[1+\left(\delta h_{b} / h_{b}\right)\right] \tan \beta-\left(\Delta h_{b} / h_{b}\right)}{1+\left(\delta h_{b} / h_{b}\right)+\left(\Delta h_{b} / h_{b}\right) \tan \beta}\right\} O_{3 i} \\
& +\operatorname{Im}\left[\frac{\left(\Delta h_{b} / h_{b}\right) \tan \beta}{1+\left(\delta h_{b} / h_{b}\right)+\left(\Delta h_{b} / h_{b}\right) \tan \beta}\right] \frac{O_{1 i}}{\cos \beta} \\
& -\operatorname{Im}\left[\frac{\left(\Delta h_{b} / h_{b}\right)}{1+\left(\delta h_{b} / h_{b}\right)+\left(\Delta h_{b} / h_{b}\right) \tan \beta}\right] \frac{O_{2 i}}{\cos \beta}, \\
& g_{H_{i} t t}^{S}=\operatorname{Re}\left[\frac{1+\left(\delta h_{t} / h_{t}\right)}{1+\left(\delta h_{t} / h_{t}\right)+\left(\Delta h_{t} / h_{t}\right) \cot \beta}\right] \frac{O_{2 i}}{\sin \beta} \\
& +\operatorname{Re}\left[\frac{\left(\Delta h_{t} / h_{t}\right)}{1+\left(\delta h_{t} / h_{t}\right)+\left(\Delta h_{t} / h_{t}\right) \cot \beta}\right] \frac{O_{1 i}}{\sin \beta} \\
& +\operatorname{Im}\left[\frac{\left(\Delta h_{t} / h_{t}\right)\left(\cot ^{2} \beta+1\right)}{1+\left(\delta h_{t} / h_{t}\right)+\left(\Delta h_{t} / h_{t}\right) \cot \beta}\right] O_{3 i} \\
& g_{H_{i} t t}^{P}=-\operatorname{Re}\left\{\frac{\left[1+\left(\delta h_{t} / h_{t}\right)\right] \cot \beta-\left(\Delta h_{t} / h_{t}\right)}{1+\left(\delta h_{t} / h_{t}\right)+\left(\Delta h_{t} / h_{t}\right) \cot \beta}\right\} O_{3 i}
\end{aligned}
$$

\footnotetext{
${ }^{6}$ Here, we have also used the fact that $b$ - and $t$-quark masses are positive, i.e. $\operatorname{Im} m_{b} \propto \operatorname{Im}\left[h_{b}+\left(\delta h_{b}\right)+\right.$ $\left.\left(\Delta h_{b}\right) \tan \beta\right]=0$ and $\operatorname{Im} m_{t} \propto \operatorname{Im}\left[h_{t}+\left(\delta h_{t}\right)+\left(\Delta h_{t}\right) \cot \beta\right]=0$.
} 


$$
\begin{aligned}
& +\operatorname{Im}\left[\frac{\left(\Delta h_{t} / h_{t}\right) \cot \beta}{1+\left(\delta h_{t} / h_{t}\right)+\left(\Delta h_{t} / h_{t}\right) \cot \beta}\right] \frac{O_{2 i}}{\sin \beta} \\
& -\operatorname{Im}\left[\frac{\left(\Delta h_{t} / h_{t}\right)}{1+\left(\delta h_{t} / h_{t}\right)+\left(\Delta h_{t} / h_{t}\right) \cot \beta}\right] \frac{O_{1 i}}{\sin \beta} .
\end{aligned}
$$

In (A.2)-(A.5), $O$ is a $3 \times 3$-dimensional mixing matrix that relates weak to mass eigenstates of Higgs bosons in the presence of CP violation [6, 8], and $\delta h_{t, b} / h_{t, b}$ and $\Delta h_{t, b} / h_{t, b}$ represent non-logarithmic threshold contributions to bottom and top Yukawa couplings [46]. As is shown in Fig. A1, the latter quantities are predominantly induced by gluino and Higgsino loops. In the presence of $\mathrm{CP}$ violation, their analytic forms are [8]

$$
\begin{aligned}
\frac{\delta h_{b}}{h_{b}} & =-\frac{2 \alpha_{s}}{3 \pi} m_{\tilde{g}}^{*} A_{b} I\left(m_{\tilde{b}_{1}}^{2}, m_{\tilde{b}_{2}}^{2},\left|m_{\tilde{g}}\right|^{2}\right)-\frac{\left|h_{t}\right|^{2}}{16 \pi^{2}}|\mu|^{2} I\left(m_{\tilde{t}_{1}}^{2}, m_{\tilde{t}_{2}}^{2},|\mu|^{2}\right), \\
\frac{\Delta h_{b}}{h_{b}} & =\frac{2 \alpha_{s}}{3 \pi} m_{\tilde{g}}^{*} \mu^{*} I\left(m_{\tilde{b}_{1}}^{2}, m_{\tilde{b}_{2}}^{2},\left|m_{\tilde{g}}\right|^{2}\right)+\frac{\left|h_{t}\right|^{2}}{16 \pi^{2}} A_{t}^{*} \mu^{*} I\left(m_{\tilde{t}_{1}}^{2}, m_{\tilde{t}_{2}}^{2},|\mu|^{2}\right), \\
\frac{\Delta h_{t}}{h_{t}} & =\frac{2 \alpha_{s}}{3 \pi} m_{\tilde{g}}^{*} \mu^{*} I\left(m_{\tilde{t}_{1}}^{2}, m_{\tilde{t}_{2}}^{2},\left|m_{\tilde{g}}\right|^{2}\right)+\frac{\left|h_{b}\right|^{2}}{16 \pi^{2}} A_{b}^{*} \mu^{*} I\left(m_{\tilde{b}_{1}}^{2}, m_{\tilde{b}_{2}}^{2},|\mu|^{2}\right), \\
\frac{\delta h_{t}}{h_{t}} & =-\frac{2 \alpha_{s}}{3 \pi} m_{\tilde{g}}^{*} A_{t} I\left(m_{\tilde{t}_{1}}^{2}, m_{\tilde{t}_{2}}^{2},\left|m_{\tilde{g}}\right|^{2}\right)-\frac{\left|h_{b}\right|^{2}}{16 \pi^{2}}|\mu|^{2} I\left(m_{\tilde{b}_{1}}^{2}, m_{\tilde{b}_{2}}^{2},|\mu|^{2}\right),
\end{aligned}
$$

where $\alpha_{s}=g_{s}^{2} /(4 \pi)$ is the $\mathrm{SU}(3)_{c}$ fine structure constant, and $I(a, b, c)$ is the one-loop function

$$
I(a, b, c)=\frac{a b \ln (a / b)+b c \ln (b / c)+a c \ln (c / a)}{(a-b)(b-c)(a-c)} .
$$

In addition, the stop and sbottom masses are given by (with $q=t, b$ )

$$
\begin{aligned}
m_{\tilde{q}_{1}\left(\tilde{q}_{2}\right)}^{2}= & \frac{1}{2}\left\{\widetilde{M}_{Q}^{2}+\widetilde{M}_{q}^{2}+2 m_{q}^{2}+T_{z}^{q} \cos 2 \beta M_{Z}^{2}\right. \\
& \left.+(-) \sqrt{\left[\widetilde{M}_{Q}^{2}-\widetilde{M}_{q}^{2}+\cos 2 \beta M_{Z}^{2}\left(T_{z}^{q}-2 Q_{q} \sin ^{2} \theta_{w}\right)\right]^{2}+4 m_{q}^{2}\left|A_{q}-R_{q} \mu^{*}\right|^{2}}\right\},
\end{aligned}
$$

where $Q_{t}\left(Q_{b}\right)=2 / 3(-1 / 3), T_{z}^{t}=-T_{z}^{b}=1 / 2, R_{t}\left(R_{b}\right)=\cot \beta(\tan \beta)$, and $\sin ^{2} \theta_{w}=$ $1-M_{W}^{2} / M_{Z}^{2}$.

It is important to remark here that only the CP-violating vertex effects on $g_{H_{i} b b}^{S}$ and $g_{H_{i} b b}^{P}$, which are proportional to $\operatorname{Im}\left[\left(\Delta h_{b} / h_{b}\right) \tan ^{2} \beta\right]$ in (A.2) and (A.3), are enhanced for moderately large values of $\tan \beta$, i.e. $20 \lesssim \tan \beta \lesssim 40$. However, for very large values of $\tan \beta$, i.e. $\tan \beta \gtrsim 40$, there is a $1 / \tan ^{2} \beta$-dependent damping factor due to CP-violating resummation effects which cancels the $\tan ^{2} \beta$-enhanced factor mentioned above. As a consequence, in the large- $\tan \beta$ limit, the coupling factors $g_{H_{i} b b}^{S}$ and $g_{H_{i} b b}^{P}$ approach a $\tan \beta$-independent constant. A related discussion is also given in Section 2. 


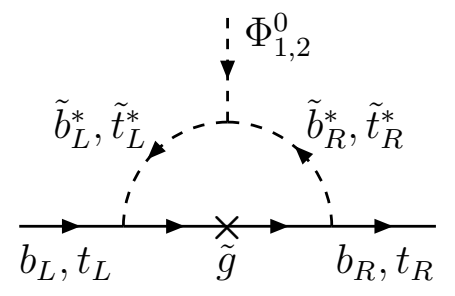

(a)

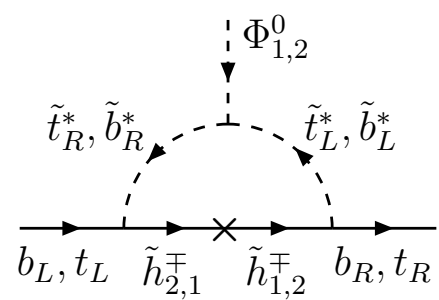

(b)

Figure A1: Effective one-loop $\Phi_{1,2}^{0} \bar{b} b$ and $\Phi_{1,2}^{0} \bar{t} t$ couplings, $\delta h_{b, t}$ and $\Delta h_{b, t}$, generated by the exchange of (a) gluinos $\tilde{g}$ and (b) Higgsinos $\tilde{h}_{1,2}^{ \pm}$.

Another important ingredient for our computation of two-loop EDMs is the diagonal effective couplings of the Higgs bosons to scalar top and bottom quarks. Taking the CPviolating Higgs-mixing effects into account, the effective Lagrangian of interest to us may be conveniently written in the form

$$
\mathcal{L}_{H \tilde{q}^{*} \tilde{q}}^{\mathrm{diag}}=\sum_{i=1}^{3} H_{i} \sum_{q=t, b}\left[v \xi_{q}^{\left(H_{i}\right)}\left(\tilde{q}_{1}^{*} \tilde{q}_{1}-\tilde{q}_{2}^{*} \tilde{q}_{2}\right)+v \zeta_{q}^{\left(H_{i}\right)}\left(\tilde{q}_{1}^{*} \tilde{q}_{1}+\tilde{q}_{2}^{*} \tilde{q}_{2}\right)\right]
$$

where

$$
\begin{aligned}
\xi_{t}^{\left(H_{i}\right)} & =\frac{2 m_{t}^{2}}{v^{2}\left(m_{\tilde{t}_{2}}^{2}-m_{\tilde{t}_{1}}^{2}\right)}\left[\operatorname{Im}\left(\mu A_{t}\right) \frac{O_{3 i}}{\sin ^{2} \beta}-\operatorname{Re}\left(\mu X_{t}\right) \frac{O_{1 i}}{\sin \beta}+\operatorname{Re}\left(A_{t}^{*} X_{t}\right) \frac{O_{2 i}}{\sin \beta}\right], \\
\xi_{b}^{\left(H_{i}\right)} & =\frac{2 m_{b}^{2}}{v^{2}\left(m_{\tilde{b}_{2}}^{2}-m_{\tilde{b}_{1}}^{2}\right)}\left[\operatorname{Im}\left(\mu A_{b}\right) \frac{O_{3 i}}{\cos ^{2} \beta}-\operatorname{Re}\left(\mu X_{b}\right) \frac{O_{2 i}}{\cos \beta}+\operatorname{Re}\left(A_{b}^{*} X_{b}\right) \frac{O_{1 i}}{\cos \beta}\right],(\mathrm{A} \\
\zeta_{t}^{\left(H_{i}\right)} & =-\frac{2 m_{t}^{2}}{v^{2}} \frac{O_{2 i}}{\sin \beta}+\mathcal{O}\left(g_{w}^{2}, g^{\prime 2}\right), \quad \zeta_{b}^{\left(H_{i}\right)}=-\frac{2 m_{b}^{2}}{v^{2}} \frac{O_{1 i}}{\cos \beta}+\mathcal{O}\left(g_{w}^{2}, g^{\prime 2}\right)
\end{aligned}
$$

with $X_{q}=A_{q}-R_{q} \mu^{*}(q=t, b)$. Although we assumed $m_{\tilde{q}_{1}}^{2}>m_{\tilde{q}_{2}}^{2}$, the effective Lagrangian (A.12) exhibits the nice feature that it is fully independent of the hierarchy of squark masses.

Finally, we present the effective couplings of the CP-mixed Higgs bosons $H_{1,2,3}$ to charginos $\chi_{1,2}^{+}$[0, 47]. These may be conveniently described by the effective Lagrangian

$$
\mathcal{L}_{H \chi^{+} \chi^{-}}=-\frac{g_{w}}{2 \sqrt{2}} \sum_{i=1}^{3} H_{i} \sum_{j, k=1,2} \bar{\chi}_{j}^{+}\left(a_{H_{i} \chi_{j}^{-} \chi_{k}^{+}}+b_{H_{i} \chi_{j}^{-} \chi_{k}^{+}} i \gamma_{5}\right) \chi_{k}^{+},
$$

where

$$
a_{H_{i} \chi_{j}^{-} \chi_{k}^{+}}=O_{1 i}\left(C_{2 j}^{R *} C_{1 k}^{L}+C_{2 k}^{R} C_{1 j}^{L *}\right)+O_{2 i}\left(C_{1 j}^{R *} C_{2 k}^{L}+C_{1 k}^{R} C_{2 j}^{L *}\right)
$$




$$
\begin{aligned}
& -i O_{3 i}\left[\sin \beta\left(C_{2 j}^{R *} C_{1 k}^{L}-C_{2 k}^{R} C_{1 j}^{L *}\right)+\cos \beta\left(C_{1 j}^{R *} C_{2 k}^{L}-C_{1 k}^{R} C_{2 j}^{L *}\right)\right], \\
b_{H_{i} \chi_{j}^{-} \chi_{k}^{+}}= & i O_{1 i}\left(C_{2 j}^{R *} C_{1 k}^{L}-C_{2 k}^{R} C_{1 j}^{L *}\right)+i O_{2 i}\left(C_{1 j}^{R *} C_{2 k}^{L}-C_{1 k}^{R} C_{2 j}^{L *}\right) \\
& +O_{3 i}\left[\sin \beta\left(C_{2 j}^{R *} C_{1 k}^{L}+C_{2 k}^{R} C_{1 j}^{L *}\right)+\cos \beta\left(C_{1 j}^{R *} C_{2 k}^{L}+C_{1 k}^{R} C_{2 j}^{L *}\right)\right] .
\end{aligned}
$$

In the above, $C^{R}$ and $C^{L}$ are $2 \times 2$ unitary matrices, which diagonalize the chargino mass matrix:

$$
M_{C}=\left(\begin{array}{cc}
m_{\widetilde{W}} & g_{w}\left\langle\phi_{2}^{0 *}\right\rangle \\
g_{w}\left\langle\phi_{1}^{0}\right\rangle & \mu
\end{array}\right),
$$

with $\left\langle\phi_{1}^{0}\right\rangle=v_{1} / \sqrt{2}$ and $\left\langle\phi_{2}^{0 *}\right\rangle=v_{2} / \sqrt{2}$, through the bi-unitary transformation

$$
C^{R \dagger} M_{C} C^{L}=\operatorname{diag}\left(m_{\chi_{1}^{+}}, m_{\chi_{2}^{+}}\right) .
$$

In (A.20), the chargino mass-eigenvalues are given by

$$
\begin{aligned}
m_{\chi_{1}^{+}\left(\chi_{2}^{+}\right)=} & \frac{1}{2}\left[\left|m_{\widetilde{W}}^{2}\right|+|\mu|^{2}+2 M_{W}^{2}\right. \\
& \left.-(+) \sqrt{\left(\left|m_{\widetilde{W}}^{2}\right|+|\mu|^{2}+2 M_{W}^{2}\right)^{2}-4\left|m_{\widetilde{W}} \mu-M_{W}^{2} \sin 2 \beta\right|^{2}}\right]
\end{aligned}
$$

while the analytic expressions for the mixing matrices $C^{L, R}$ are quite lengthy in the presence of $\mathrm{CP}$ violation, and will not be presented here; they can be computed using standard techniques [7].

For completeness, we give the corresponding effective couplings of the would-be Goldstone boson $G^{0}$ to charginos $\chi_{1,2}^{+}$:

$$
\begin{aligned}
& a_{G^{0} \chi_{j}^{-} \chi_{k}^{+}}=i \cos \beta\left(C_{2 j}^{R *} C_{1 k}^{L}-C_{2 k}^{R} C_{1 j}^{L *}\right)-i \sin \beta\left(C_{1 j}^{R *} C_{2 k}^{L}-C_{1 k}^{R} C_{2 j}^{L *}\right), \\
& b_{G^{0} \chi_{j}^{-} \chi_{k}^{+}}=-\cos \beta\left(C_{2 j}^{R *} C_{1 k}^{L}+C_{2 k}^{R} C_{1 j}^{L *}\right)+\sin \beta\left(C_{1 j}^{R *} C_{2 k}^{L}+C_{1 k}^{R} C_{2 j}^{L *}\right) .
\end{aligned}
$$

A non-trivial consistency check for the correctness of our analytic results is the vanishing of the diagonal scalar couplings of the $G^{0}$ boson to charginos, i.e. $a_{G^{0} \chi_{j}^{-}} \chi_{j}^{+}=0$. 


\section{References}

[1] G.F. Giudice and S. Dimopoulos, Phys. Lett. B357 (1995) 573; G. Dvali and A. Pomarol, Phys. Rev. Lett. 77 (1996) 3728; A.G. Cohen, D.B. Kaplan and A.E. Nelson, Phys. Lett. B388 (1996) 588; P. Binétruy and E. Dudas, Phys. Lett. B389 (1996) 503.

[2] D. Chang, W.-Y. Keung and A. Pilaftsis, Phys. Rev. Lett. 82 (1999) 900 and 83 (1999) $3972(\mathrm{E})$.

[3] A. Pilaftsis, Phys. Lett. B471 (1999) 174; D. Chang, W.-F. Chang and W.-Y. Keung, Phys. Lett. B478 (2000) 239.

[4] S.M. Barr and A. Zee, Phys. Rev. Lett. 65 (1990) 21.

[5] A. Pilaftsis, Phys. Rev. D58 (1998) 096010 and Phys. Lett. B435 (1998) 88.

[6] A. Pilaftsis and C.E.M. Wagner, Nucl. Phys. B553 (1999) 3; D.A. Demir, Phys. Rev. D60 (1999) 055006; S.Y. Choi, M. Drees and J.S. Lee, Phys. Lett. B481 (2000) 57; G.L. Kane and L.-T. Wang, Phys. Lett. B488 (2000) 383; S.Y. Choi, K. Hagiwara and J.S. Lee, Phys. Rev. D64 (2001) 032004; Phys. Lett. B529 (2002) 212, S. Heinemeyer, Eur. Phys. J. C22 (2001) 521; T. Ibrahim and P. Nath, hep-ph/0204092; S.W. Ham, S.K. Oh, E.J. Yoo, C.M. Kim and D. Son, hep-ph/0205244.

[7] T. Ibrahim and P. Nath, Phys. Rev. D63 (2001) 035009.

[8] M. Carena, J. Ellis, A. Pilaftsis and C.E.M. Wagner, Nucl. Phys. B586 (2000) 92.

[9] M. Carena, J. Ellis, A. Pilaftsis and C.E.M. Wagner, Phys. Lett. B495 (2000) 155.

[10] M. Carena, J. Ellis, A. Pilaftsis and C.E.M. Wagner, Nucl. Phys. B625 (2002) 345.

[11] For phenomenological applications of radiative Higgs-sector CP violation in the MSSM, see: S.Y. Choi and M. Drees, Phys. Rev. Lett. 81 (1998) 5509; S. Baek and P. Ko, Phys. Rev. Lett. 83 (1999) 488; W. Bernreuther, A. Brandenburg and M. Flesch, hep-ph/9812387; K.S. Babu, C. Kolda, J. March-Russell and F. Wilczek, Phys. Rev. D59 (1999) 016004; B. Grzadkowski, J.F. Gunion and J. Kalinowski, Phys. Rev. D60 (1999) 075011; S.Y. Choi, M. Guchait, H.S. Song and W.Y. Song, Phys. Lett. B483 (2000) 168; P. Osland, Acta Phys. Polon. B30 (1999) 1967; T. Han, T. Huang, Z.H. Lin, J.X. Wang and X. Zhang, Phys. Rev. D61 (2000) 015006; B. Grzadkowski and 
J. Pliszka, Phys. Rev. D60 (1999) 115018; S.Y. Choi and J.S. Lee, Phys. Rev. D61 (2000) 015003; C.-S. Huang and L. Wei, Phys. Rev. D61 (2000) 116002; K. Freese and P. Gondolo, hep-ph/9908390; S.Y. Choi and J.S. Lee, Phys. Rev. D61 (2000) 115002; A. Dedes and S. Moretti, Phys. Rev. Lett. 84 (2000) 22; Nucl. Phys. B576 (2000) 29; M.S. Berger, Phys. Rev. Lett. 87 (2001) 131801; D.A. Demir, Nucl. Phys. Proc. Suppl. 101 (2001) 431; S.W. Ham, S.K. Oh, E.J. Yoo and H.K. Lee, J. Phys. G27 (2001) 1; A.G. Akeroyd and A. Arhrib, Phys. Rev. D64 (2001) 095018; M. Boz and N.K. Pak, Phys. Rev. D65 (2002) 075014; A. Arhrib, D.K. Ghosh and O.C.W. Kong, Phys. Lett. B537 (2002) 217; E. Christova, S. Fichtinger, S. Kraml and W. Majerotto, Phys. Rev. D65 (2002) 094002; hep-ph/0205227; C.-H. Chen, hep-ph/0206143; M.N. Dubinin and A.V. Semenov, hep-ph/0206205; A. Bartl, K. Hidaka, T. Kernreiter and W. Porod, hep-ph/0207186.

[12] V.A. Kuzmin, V.A. Rubakov and M.E. Shaposhnikov, Phys. Lett. B155 (1985) 36.

[13] For recent studies, see: M. Carena, M. Quirós and C.E.M. Wagner, Nucl. Phys. B524 (1998) 3; M. Laine and K. Rummukainen, Phys. Rev. Lett. 80 (1998) 5259; Nucl. Phys. B535 (1998) 423; Nucl. Phys. B597 (2001) 23; K. Funakubo, Prog. Theor. Phys. 102 (1999) 389; J. Grant and M. Hindmarsh, Phys. Rev. D59 (1999) 116014; M. Losada, Nucl. Phys. B537 (1999) 3; Nucl. Phys. B569 (2000) 125; S. Davidson, M. Losada and A. Riotto, Phys. Rev. Lett. 84 (2000) 4284; A.B. Lahanas, V.C. Spanos and V. Zarikas, Phys. Lett. B472 (2000) 119; N. Rius and V. Sanz, Nucl. Phys. B570 (2000) 155; J.M. Cline, M. Joyce and K. Kainulainen, JHEP 0007 (2000) 018; [Erratum:hepph/0110031]; S.J. Huber, P. John and M.G. Schmidt, Eur. Phys. J. C20 (2001) 695; H. Murayama and A. Pierce, hep-ph/0201261.

[14] M. Carena, J.M. Moreno, M. Quirós, M. Seco and C.E.M. Wagner, Nucl. Phys. B599 (2001) 158; and work in progress.

[15] P.G. Harris et al., Phys. Rev. Lett. 82 (1999) 904.

[16] B.C. Regan, E.D. Commins, C.J. Schmidt and D. DeMille, Phys. Rev. Lett. 88 (2002) 071805.

[17] S. Barr, Phys. Rev. Lett. 68 (1992) 1822; Int. J. Mod. Phys. A8 (1993) 209.

[18] W. Fischler, S. Paban and S. Thomas, Phys. Lett. B289 (1992) 373.

[19] I.B. Khriplovich and S.K. Lamoreaux, CP Violation Without Strangeness (Springer, New York, 1997). 
[20] Y.K. Semertzidis et al., Letter of Intent to BNL (Spring of 2000, and Fall of 1997); "Sensitive Search for a Permanent Muon Electric Dipole Moment" hep-ph/0012087. For recent theoretical studies, see K.S. Babu, B. Dutta and R.N. Mohapatra, Phys. Rev. Lett. 85 (2000) 5064; T. Ibrahim and P. Nath, Phys. Rev. D64 (2001) 093002; J.L. Feng, K.T. Matchev and Y. Shadmi, Nucl. Phys. B613 (2001) 366; J.R. Ellis, J. Hisano, M. Raidal and Y. Shimizu, Phys. Lett. B528 (2002) 86.

[21] K.F. Smith et al., Phys. Lett. B234 (1990) 191.

[22] P.G. Harris et al., Phys. Rev. Lett. 82 (1999) 904.

[23] S.K. Lamoreaux and R. Golub, Phys. Rev. D61 (2000) 051301.

[24] M. Pospelov and A. Ritz, Phys. Rev. Lett. 83 (1999) 2526; Nucl. Phys. B573 (2000) 177.

[25] S.A. Abel, S. Khalil and O. Lebedev, Nucl. Phys. B606 (2001) 151.

[26] M.V. Romalis, W.C. Griffith and E.N. Fortson, Phys. Rev. Lett. 86 (2001) 2505.

[27] T. Falk, K.A. Olive, M. Pospelov and R. Roiban, Nucl. Phys. B60 (1999) 3.

[28] A. Ritz, private communication.

[29] S. Weinberg, Phys. Rev. Lett. 63 (1989) 2333.

[30] J. Dai, H. Dykstra, R.G. Leigh, S. Paban, and D.A. Dicus, Phys. Lett. B237 (1990) 216 and B242 (1990) 547 (E).

[31] T. Ibrahim and P. Nath, Phys. Lett. B418 (1998) 98, Phys. Rev. D57 (1998) 478, D58 (1998) 019901 (E), Phys. Rev. D58 (1998) 111301; Phys. Rev. D61 (2000) 093004; M. Brhlik, G.J. Good and G.L. Kane, Phys. Rev. D59 (1999) 115004; M. Brhlik, L. Everett, G.L. Kane and J. Lykken, Phys. Rev. Lett. 83 (1999) 2124; Phys. Rev. D62 (2000) 035005; S. Abel, S. Khalil and O. Lebedev, Phys. Rev. Lett. 86 (2001) 5850.

[32] J. Ellis, S. Ferrara and D.V. Nanopoulos, Phys. Lett. B114 (1982) 231; W. Buchmüller and D. Wyler, Phys. Lett. B121 (1983) 321; J. Polchinski and M. Wise, Phys. Lett. B125 (1983) 393; F. del Aguila, M. Gavela, J. Grifols and A. Mendez, Phys. Lett. B126 (1983) 71; D.V. Nanopoulos and M. Srednicki, Phys. Lett. B128 (1983) 61; T. Falk, K.A. Olive and M. Srednicki, Phys. Lett. B354 (1995) 99; M. Dugan, B. Grinstein and L. Hall, Nucl. Phys. B255 (1985) 413; R. Garisto and J.D. Wells, Phys. Rev. D55 (1997) 1611. 
[33] P. Nath, Phys. Rev. Lett. 66 (1991) 2565; Y. Kizukuri and N. Oshimo, Phys. Rev. D46 (1992) 3025.

[34] For recent analyses of one-loop EDM effects, see S. Pokorski, J. Rosiek and C.A. Savoy, Nucl. Phys. B570 (2000) 81; E. Accomando, R. Arnowitt and B. Dutta, Phys. Rev. D61 (2000) 115003; A. Bartl, T. Gajdosik, W. Porod, P. Stockinger and H. Stremnitzer, Phys. Rev. D60 (1999) 073003.

[35] In this respect, our analysis of EDM constraints on a CP-violating MSSM Higgs sector is complementary to the one presented by T. Ibrahim, Phys. Rev. D64 (2001) 035009.

[36] A. Pilaftsis, Phys. Rev. D62 (2000) 016007.

[37] T.H. West, Phys. Rev. D50 (1994) 7; T. Kadoyoshi and N. Oshimo, Phys. Rev. D55 (1997) 1481.

[38] In our numerical analysis, we employ the Fortran code cph+.f available at http://home.cern.ch/p/pilaftsi/www/. The code is based on a recent RGimproved diagrammatic calculation of Higgs-boson pole masses in the MSSM with explicit CP violation [10].

[39] For recent two-loop studies of an effective CP-conserving Higgs potential in the MSSM, see: M. Carena, H.E. Haber, S. Heinemeyer, W. Hollik, C.E.M. Wagner and G. Weiglein, Nucl. Phys. B580 (2000) 29; J.R. Espinosa and R.J. Zhang, JHEP 0003 (2000) 026; M. Carena, S. Mrenna and C.E.M. Wagner, Phys. Rev. D62 (2000) 055008; J.R. Espinosa and I. Navarro, Nucl. Phys. B615 (2001) 82; A. Brignole, G. Degrassi, P. Slavich and F. Zwirner, Nucl. Phys. B631 (2002) 195; hep-ph/0206101; S.P. Martin, hep-ph/0206136.

[40] O. Lebedev and M. Pospelov, hep-ph/0204359.

[41] D. Chang, W.-F. Chang and W.-Y. Keung, hep-ph/0205084 [v2, 4 June 2002].

[42] M.A. Shifman, A.L. Vainshtein and V.I. Zakharov, Phys. Lett. B78 (1978) 443; see also J. Ellis, M.K. Gaillard and D.V. Nanopoulos, Nucl. Phys. B106 (1976) 292; S. Dawson and H.E. Haber, Int. J. Mod. Phys. A7 (1992) 107.

[43] OPAL Collaboration, "Interpretation of the Search for Neutral Higgs Bosons from $\sqrt{s}=91 \mathrm{GeV}$ to $\sqrt{s}=209 \mathrm{GeV}$ in a CP-Violating MSSM Scenario," OPAL Physics Note PN505, available from http://opal.web.cern.ch/Opal/pubs/ physnote/html/pn505.html. 
[44] M. Carena, J. Ellis, S. Mrenna, A. Pilaftsis and C.E.M. Wagner, work in progress.

[45] D. Chang, W.-F. Chang, C.-H. Chou and W.-Y. Keung, Phys. Rev. D63 (2001) 091301; A. Dedes and H.E. Haber, JHEP 0105 (2001) 006.

[46] E. Ma, Phys. Rev. D39 (1989) 1922; R. Hempfling, Phys. Rev. D49 (1994) 6168; L.J. Hall, R. Rattazzi and U. Sarid, Phys. Rev. D50 (1994) 7048; T. Blazek, S. Raby and S. Pokorski, Phys. Rev. D52 (1995) 4151; M. Carena, M. Olechowski, S. Pokorski and C.E.M. Wagner, Nucl. Phys. B426 (1994) 269; S. Heinemeyer, W. Hollik and G. Weiglein, Phys. Lett. B440 (1998) 296; Eur. Phys. J. C9 (1999) 343; F. Borzumatti, G. Farrar, N. Polonsky and S. Thomas, Nucl. Phys. B555 (1999) 53; J.R. Espinosa and R.J. Zhang, Nucl. Phys. B586 (2000) 3.

[47] S.Y. Choi, M. Drees, J.S. Lee and J. Song, hep-ph/0204200. 


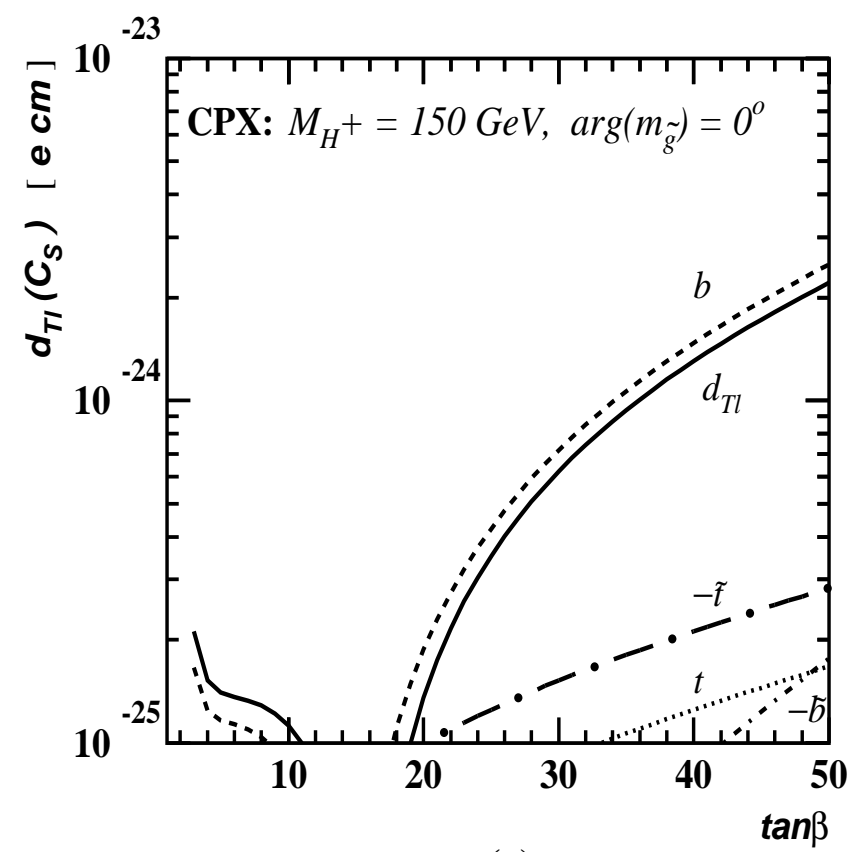

(a)

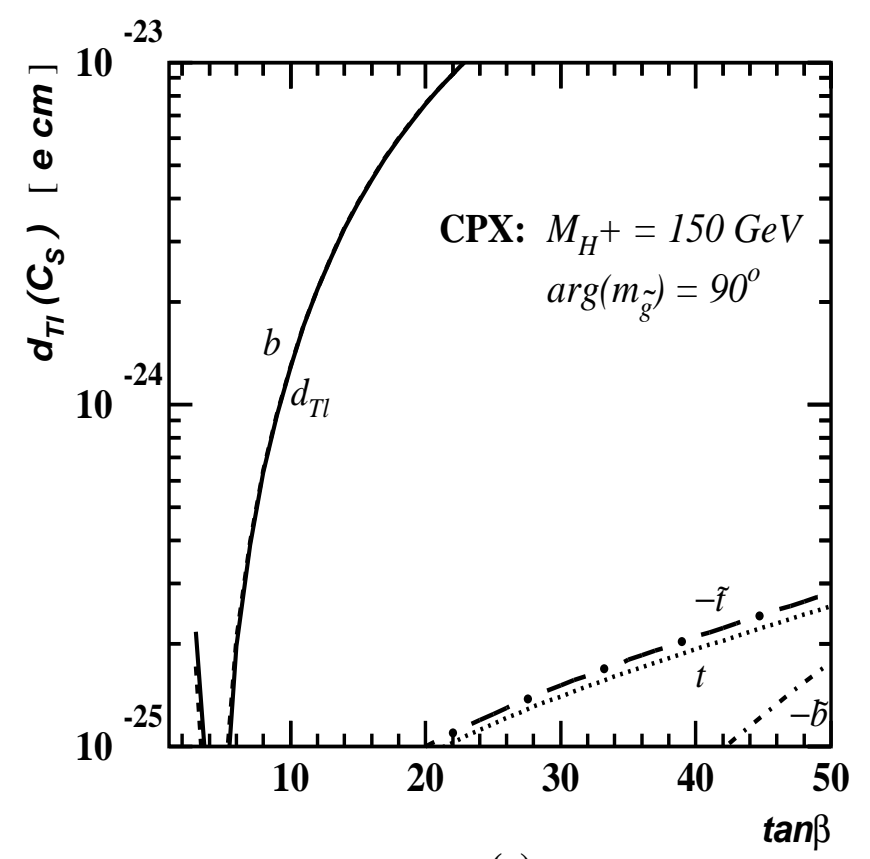

(c)

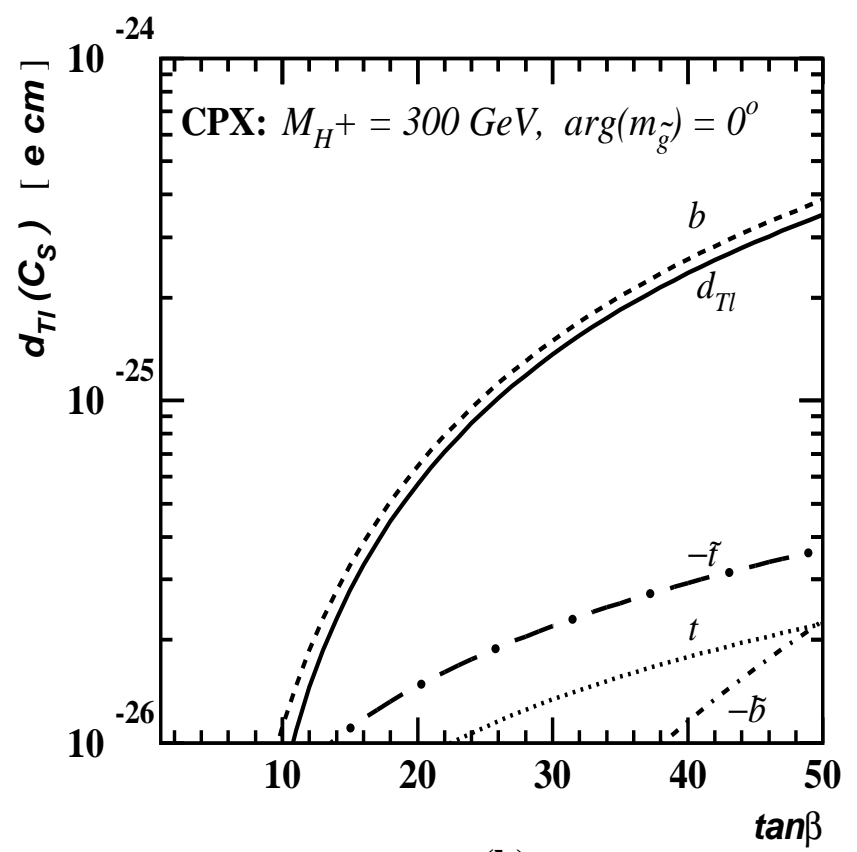

(b)

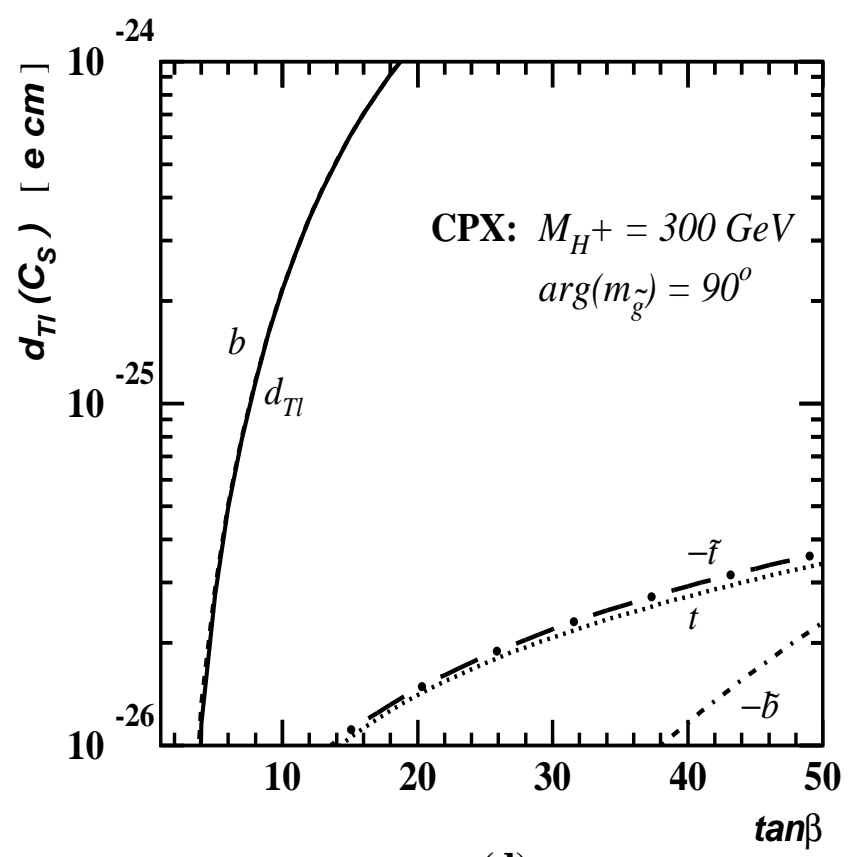

(d)

Figure 3: Numerical estimates of ${ }^{205} \mathrm{Tl} E D M d_{\mathrm{Tl}}$ induced by the CP-odd electron-nucleon operator $C_{S}$ as functions of $\tan \beta$, in four selected CPX scenarios with $M_{\mathrm{SUSY}}=1 \mathrm{TeV}$. The values of the CPX parameters are given in (4.1). The individual $b, \tilde{t}, t, \tilde{b}$ contributions to $d_{\mathrm{Tl}}\left(C_{S}\right)$, along with their relative signs, are also displayed. 


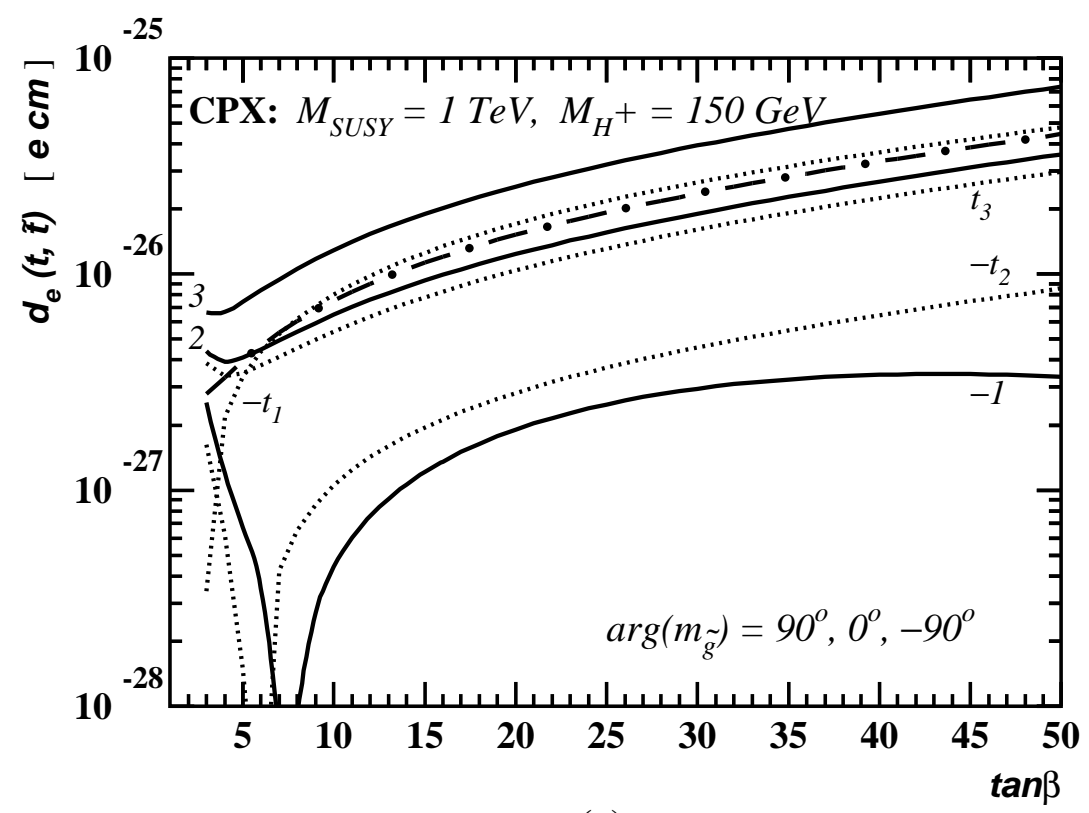

(a)

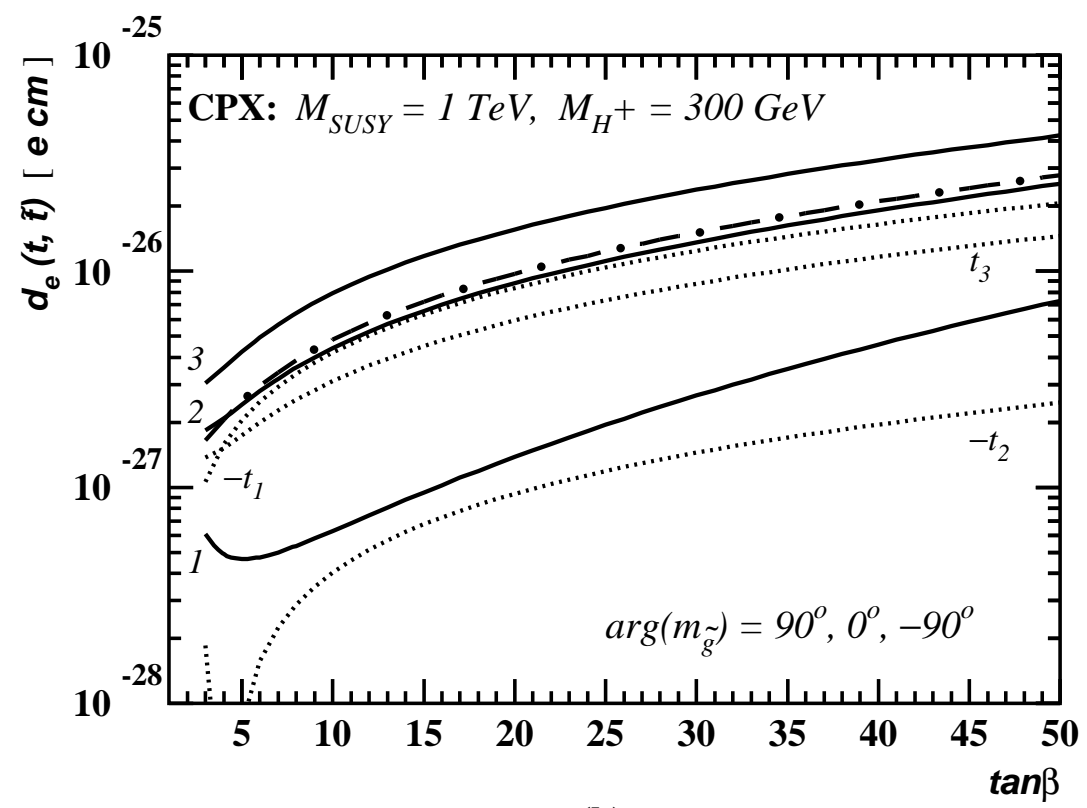

(b)

Figure 4: Numerical estimates of resummed Higgs-boson two-loop effects on $d_{e}$, induced by $t, b$ - quarks and $\tilde{t}, \tilde{b}$-squarks, as functions of $\tan \beta$, in two variants of the CPX scenario, with (a) $M_{H^{+}}=150 \mathrm{GeV}$ and (b) $M_{H^{+}}=300 \mathrm{GeV}$. The long-dash-dotted lines indicate the stop/sbottom contributions to $d_{e}$. The dotted lines $t_{1,2,3}$ correspond to top/bottom contributions, for $\arg \left(m_{\tilde{g}}\right)=90^{\circ}, 0^{\circ},-90^{\circ}$, respectively. Likewise, the solid lines 1,2,3 give the sum of all the aforementioned contributions to $d_{e}$ for the same values of gluino phases. Contributions to $d_{e}$ that are denoted with a minus sign are negative. 

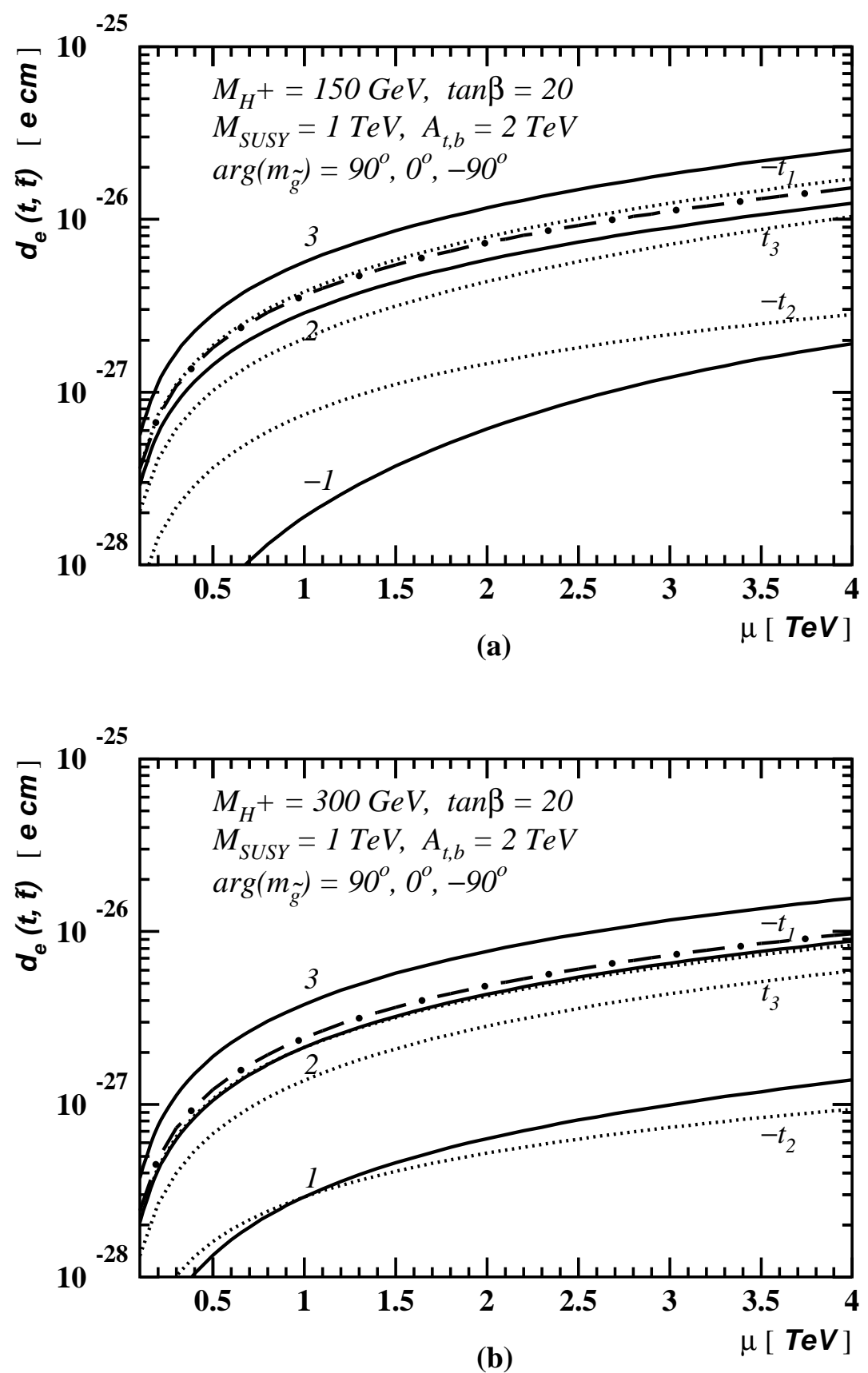

Figure 5: Numerical values of resummed Higgs-boson two-loop effects on $d_{e}$, induced by $t, b$ - quarks and $\tilde{t}, \tilde{b}$-squarks, as functions of $\mu$, in two variants of the CPX scenario, with $\tan \beta=20$, and (a) $M_{H^{+}}=150 \mathrm{GeV}$ and (b) $M_{H^{+}}=300 \mathrm{GeV}$. The meaning of the different line types is identical to that of Fig. 因. For $A_{t, b}=0$, the long-dash-dotted line disappears and so the solid lines collapse to the dotted ones. 


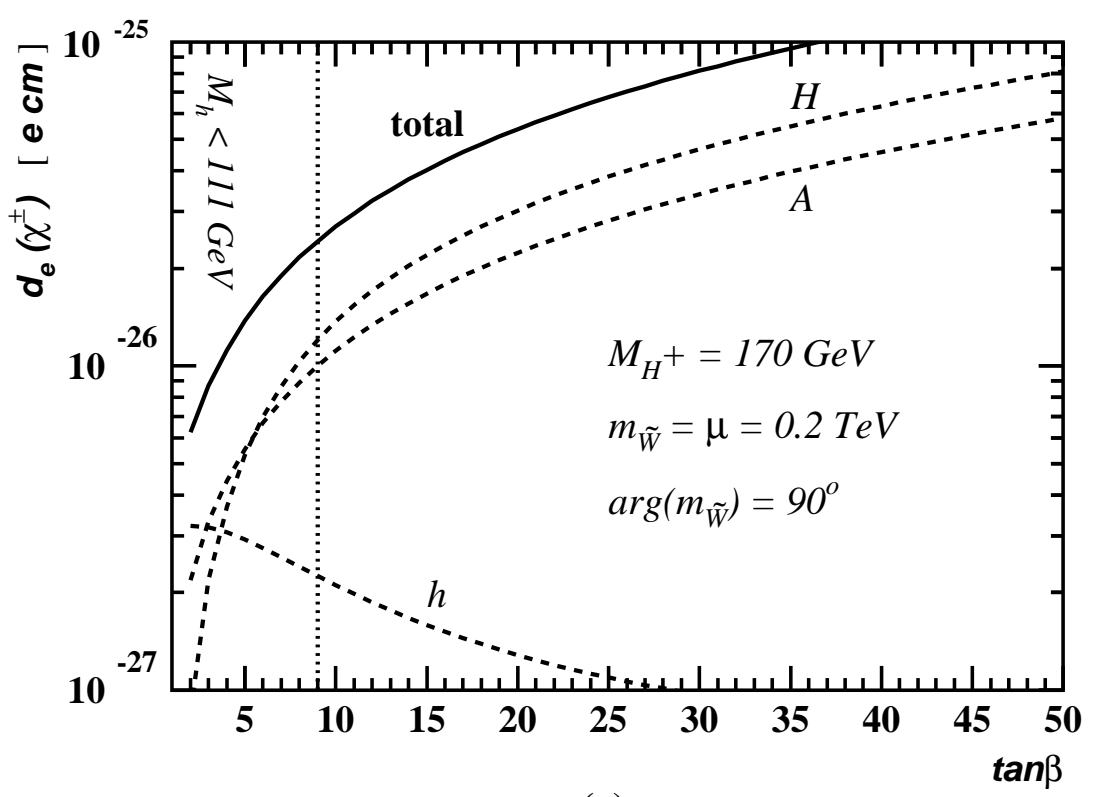

(a)

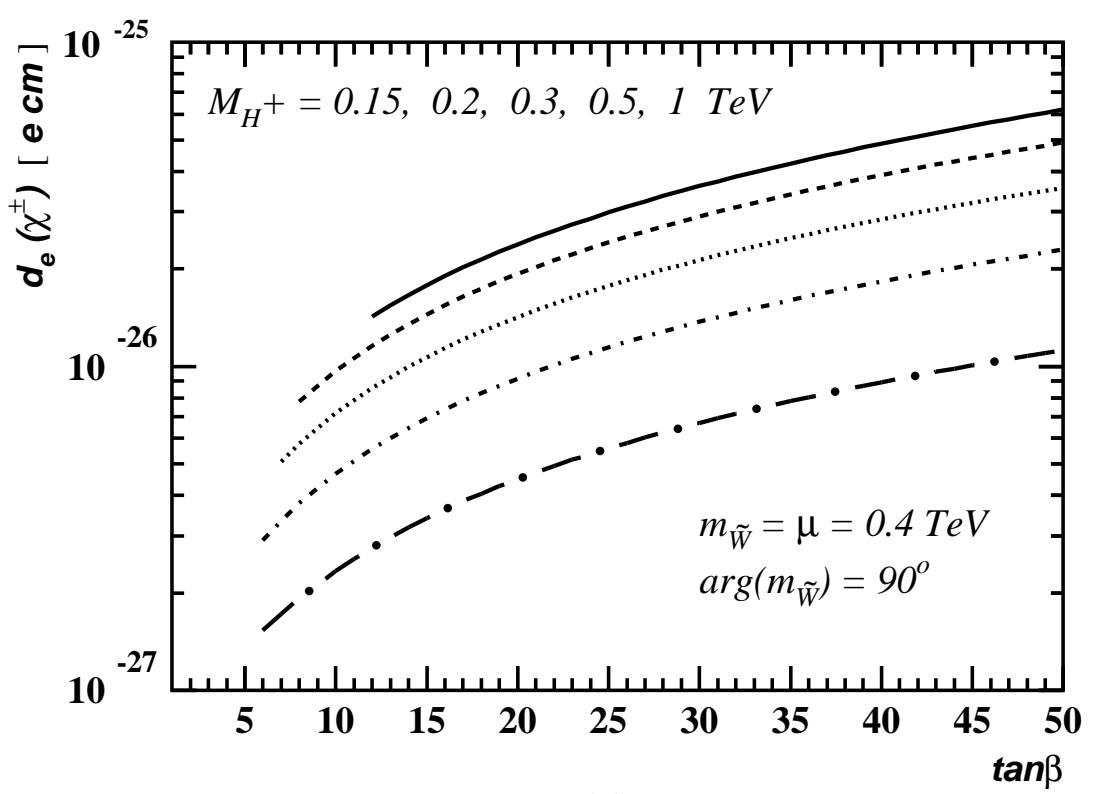

(b)

Figure 6: $d_{e}$ versus $\tan \beta$ in a scenario favoured by electroweak baryogenesis, with MSSM parameters $\widetilde{M}_{Q}=\widetilde{M}_{D}=3 \mathrm{TeV}, \widetilde{M}_{U}=0, A_{t, b}=1.8 \mathrm{Te} V, m_{\tilde{g}}=3 \mathrm{Te} V$ and $\arg \left(A_{t, b}\right)=$ $\arg \left(m_{\tilde{g}}\right)=0^{\circ}$. In (a), $M_{H^{+}}=170 \mathrm{GeV}$ is used, corresponding to $M^{\prime} A^{\prime} \approx 150 \mathrm{GeV}$, and $m_{\widetilde{W}}=\mu=0.2 \mathrm{TeV}$ and $\arg \left(m_{\widetilde{W}}\right)=90^{\circ}$. Also displayed are the individual ' $h$ ', ' $H$ ', 'A' contributions to $d_{e}$ and the LEP excluded region from direct Higgs-boson searches. In (b), numerical values are shown for $M_{H^{+}}=150 \mathrm{GeV}$ (solid), $200 \mathrm{GeV}$ (dashed), $300 \mathrm{GeV}$ (dotted), $500 \mathrm{GeV}$ (dash-dotted) and $1 \mathrm{TeV}$ (long-dash-dotted), in a scenario with $m_{\widetilde{W}}=\mu=0.4 \mathrm{TeV}$ and $\arg \left(m_{\widetilde{W}}\right)=90^{\circ}$. 


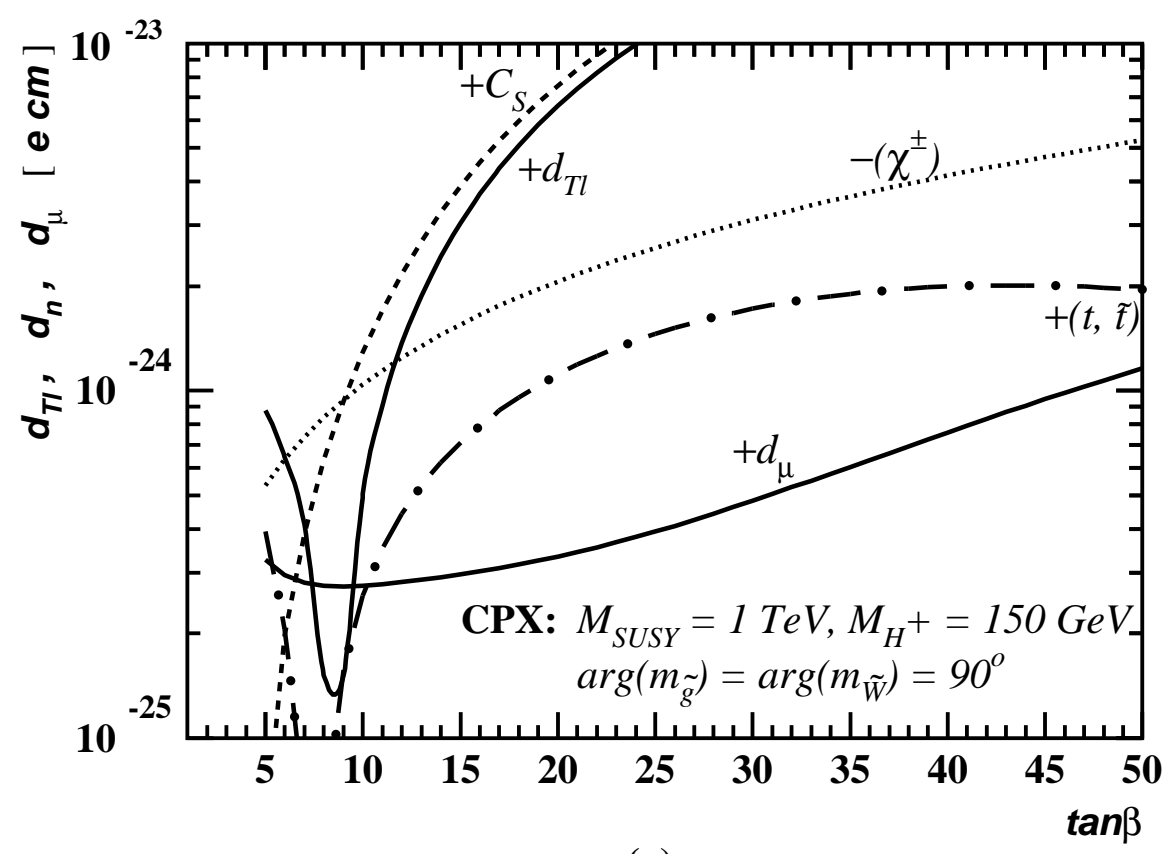

(a)

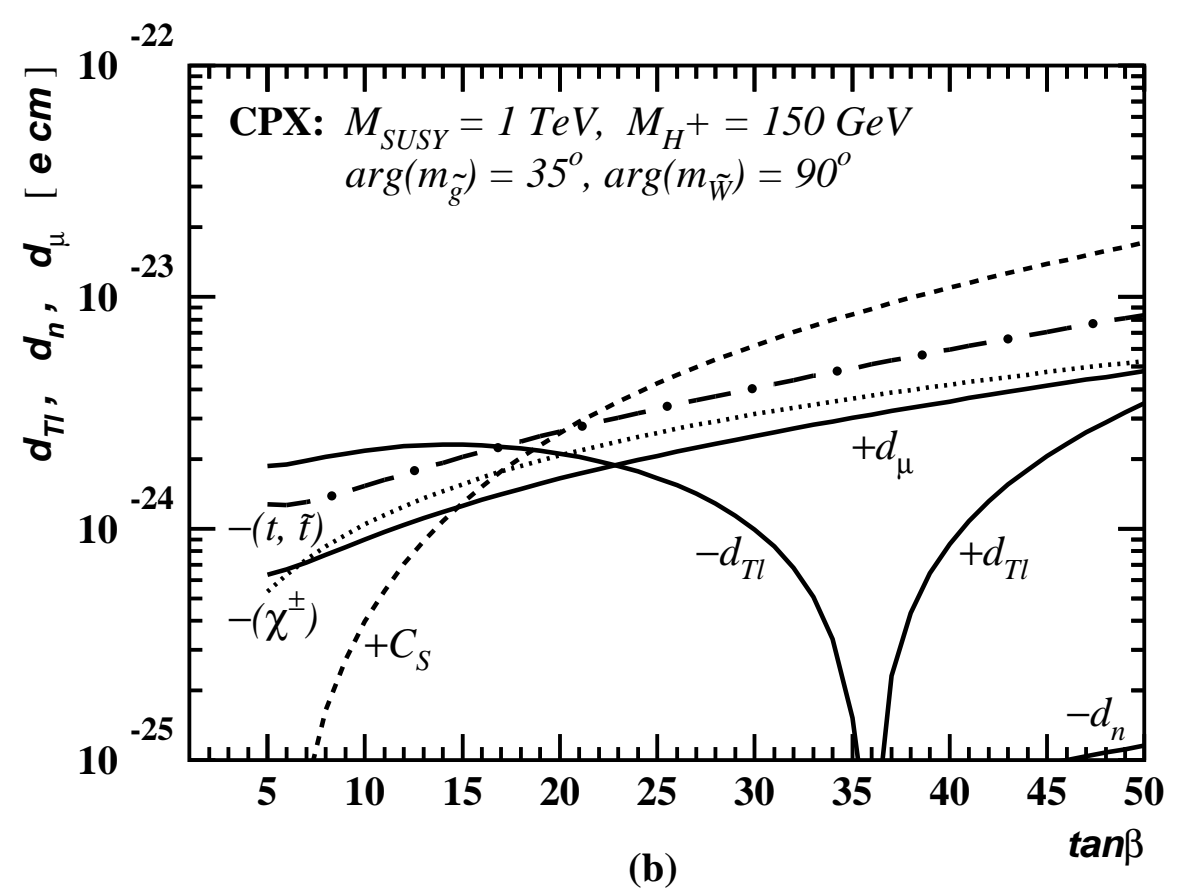

Figure 7: $E D M$ s of $d_{\mathrm{Tl}}, d_{n}$ and $d_{\mu}$ as functions of $\tan \beta$ in two versions of the CPX scenario: (a) $\arg \left(m_{\tilde{g}}\right)=\arg \left(m_{\widetilde{W}}\right)=90^{\circ}$, and $(b) \arg \left(m_{\tilde{g}}\right)=35^{\circ}, \arg \left(m_{\widetilde{W}}\right)=90^{\circ}$. Also shown are the different contributions, along with their relative signs, to $d_{\mathrm{Tl}}$ from top/stop (long-dashdotted) and chargino (dotted) Higgs-boson two-loop graphs, as well as from the CP-odd electron-nucleon coupling $C_{S}$ (dashed). 

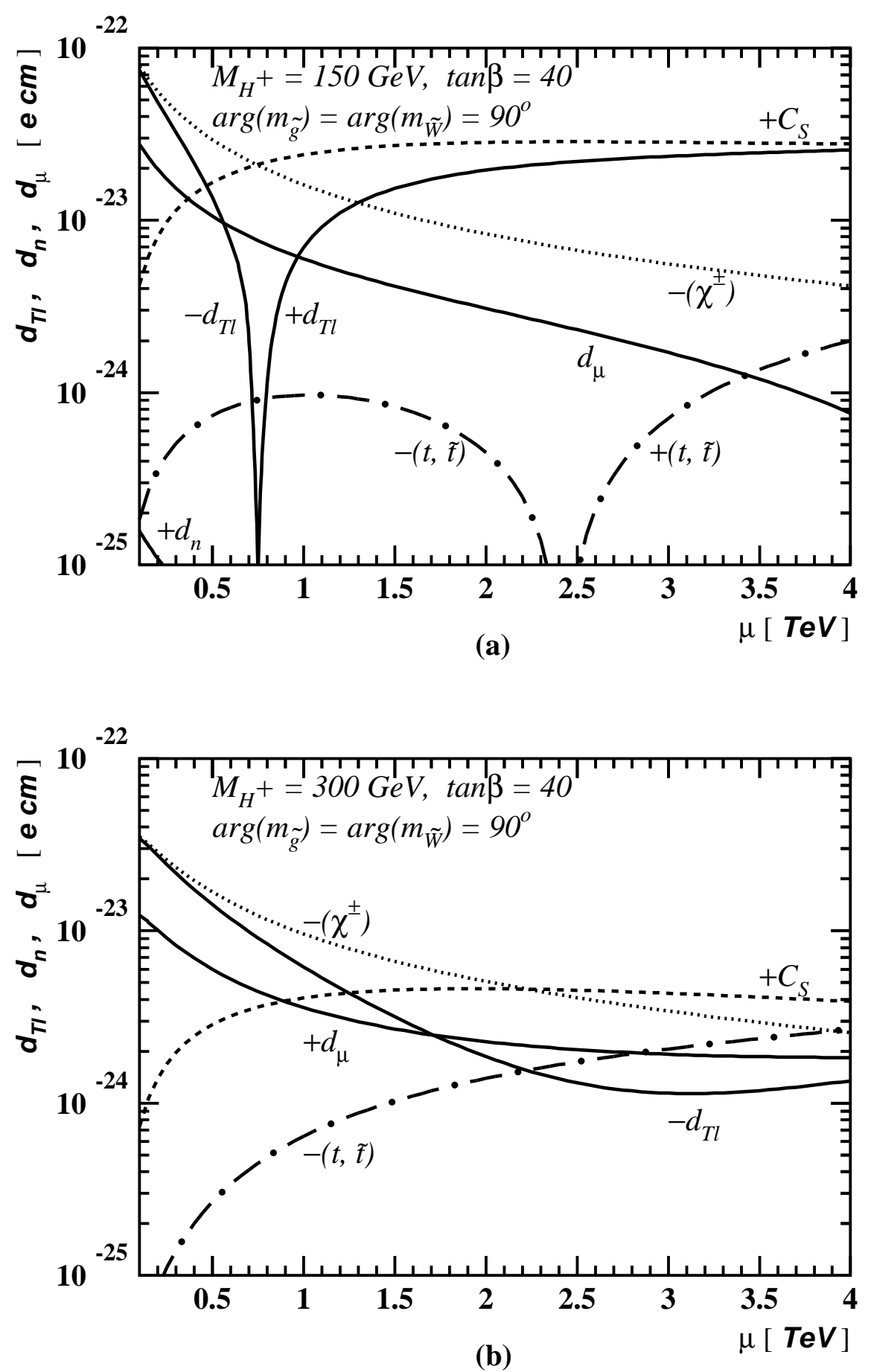

Figure 8: Numerical values of $d_{\mathrm{Tl}}, d_{n}$ and $d_{\mu}$ as functions of $\mu$ for two large-tan $\beta$ scenarios, with $\tan \beta=40, M_{\mathrm{SUSY}}=1 \mathrm{TeV}, m_{\tilde{g}}=1 \mathrm{TeV}, m_{\widetilde{W}}=m_{\widetilde{B}}=0.3 \mathrm{TeV}, \arg \left(m_{\tilde{g}}\right)=$ $\arg \left(m_{\widetilde{W}}\right)=90^{\circ}, A_{t, b}=2 \mathrm{TeV}, \arg \left(A_{t, b}\right)=90^{\circ}:$ (a) $M_{H^{+}}=150 \mathrm{GeV}$; (b) $M_{H^{+}}=300 \mathrm{GeV}$. In analogy with Fig. 0 , the individual contributions to $d_{\mathrm{Tl}}$ due to top/stop and chargino two loop graphs and due to the $C_{S}$ operator are also shown. 